\title{
ASYMPTOTIC EXPANSIONS OF LEGENDRE SERIES COEFFICIENTS FOR FUNCTIONS WITH INTERIOR AND ENDPOINT SINGULARITIES
}

\author{
AVRAM SIDI
}

Abstract. Let $\sum_{n=0}^{\infty} e_{n}[f] P_{n}(x)$ be the Legendre expansion of a function $f(x)$ on $(-1,1)$. In an earlier work [A. Sidi, Asymptot. Anal., 65 (2009), pp. $175-190]$, we derived asymptotic expansions as $n \rightarrow \infty$ for $e_{n}[f]$, assuming that $f \in C^{\infty}(-1,1)$, but may have arbitrary algebraic-logarithmic singularities at one or both endpoints $x= \pm 1$. In the present work, we extend this study to functions $f(x)$ that are infinitely differentiable on $[0,1]$, except at finitely many points $x_{1}, \ldots, x_{m}$ in $(-1,1)$ and possibly at one or both of the endpoints $x_{0}=1$ and $x_{m+1}=-1$, where they may have arbitrary algebraic singularities, including finite jump discontinuities. Specifically, we assume that, for each $r$, $f(x)$ has asymptotic expansions of the form

$$
f(x) \sim \sum_{s=0}^{\infty} W_{r s}^{( \pm)}\left|x-x_{r}\right|^{\delta_{r s}^{( \pm)}} \quad \text { as } x \rightarrow x_{r} \pm,
$$

where $W_{r s}^{( \pm)}$and $\delta_{r s}^{( \pm)}$are, in general, complex and $\Re \delta_{r s}^{( \pm)}>-1$. We derive the full asymptotic expansion of $e_{n}[f]$ as $n \rightarrow \infty$ for this very general behavior of $f(x)$. In the special case where $\delta_{r s}^{( \pm)}=\sigma_{r}^{( \pm)}+s, 1 \leq r \leq m$, and $\delta_{0 s}^{(-)}=\alpha+s$ and $\delta_{m+1, s}^{(+)}=\beta+s$, this expansion reduces to

$$
\begin{aligned}
e_{n}[f] \sim \sum_{r=1}^{m} & \left\{e^{\mathrm{i} \widehat{n} \theta_{r}}\left[\sum_{s=0}^{\infty} \frac{a_{r s}^{(+)}}{\widehat{n}^{\sigma_{r}^{(+)}}+s+1 / 2}+\sum_{s=0}^{\infty} \frac{a_{r s}^{(-)}}{\widehat{n}^{\sigma_{r}^{(-)}+s+1 / 2}}\right]\right. \\
+ & e^{-\mathrm{i} \widehat{n} \theta_{r}}\left[\sum_{s=0}^{\infty} \frac{\widehat{a}_{r s}^{(+)}}{\widehat{n}^{\sigma_{r}^{(+)}}+s+1 / 2}+\sum_{\substack{s=0 \\
\widehat{n}^{\sigma_{r}^{(-)}+s+1 / 2}}}^{\infty} \frac{\widehat{a}_{r s}^{(-)}}{\infty}\right] \\
+ & \sum_{\substack{s=0 \\
\alpha \notin \mathbb{Z}^{+}}}^{\infty} \frac{A_{s}}{\widehat{n}^{2(\alpha+s+1 / 2)}}+(-1)^{n} \sum_{\substack{s=0 \\
\beta \notin \mathbb{Z}^{+}}}^{\infty} \frac{B_{s}}{\widehat{n}^{2(\beta+s+1 / 2)}} \text { as } n \rightarrow \infty
\end{aligned}
$$

where $\theta_{r}=\cos ^{-1} x_{r}, \widehat{n}=n+1 / 2, \mathbb{Z}^{+}=\{0,1,2, \ldots\}$, and $a_{r s}^{( \pm)}, \widehat{a}_{r s}^{( \pm)}, A_{s}$, and $B_{s}$ are constants independent of $n$. In the course of this study, we also derive a full asymptotic expansion as $n \rightarrow \infty$ for integrals of the form $\int_{c}^{d} f(x) P_{n}(x) d x$ where $[c, d] \in(-1,1)$ and $f \in C^{\infty}[c, d]$ or $f \in C^{\infty}(c, d)$ but may have arbitrary algebraic singularities at $x=c$ and/or $x=d$.

Received by the editor March 17, 2010 and, in revised form, May 25, 2010.

2000 Mathematics Subject Classification. Primary 40A05, 40A10, 41A58, 41A60, 42C10.

Key words and phrases. Legendre polynomials, Legendre series, interior singularities, endpoint singularities, asymptotic expansions.

This research was supported in part by the United States-Israel Binational Science Foundation grant no. 2008399.

(C)2010 American Mathematical Society Reverts to public domain 28 years from publication 


\section{INTRODUCTION}

Let $\sum_{n=0}^{\infty} e_{n}[f] P_{n}(x)$ be the Legendre series of a function $f(x)$ on $(-1,1)$. Here $P_{n}(x)$ is the $n$th Legendre polynomial standardized such that $P_{n}(1)=1$, so that

$$
\int_{-1}^{1} P_{m}(x) P_{n}(x) d x=\frac{1}{n+1 / 2} \delta_{m, n}, \quad m, n=0,1, \ldots,
$$

and hence

$$
e_{n}[f]=(n+1 / 2) \int_{-1}^{1} f(x) P_{n}(x) d x, \quad n=0,1, \ldots .
$$

In a recent paper [12, we derived asymptotic expansions as $n \rightarrow \infty$ for $e_{n}[f]$, assuming that $f \in C^{\infty}(-1,1)$, but may have arbitrary algebraic-logarithmic singularities at one or both endpoints $x= \pm 1$. In the present work, we extend the results of Sidi 12 to the case in which $f(x)$ may have arbitrary algebraic singularities, including finite jump discontinuities, at finitely many points in $(-1,1)$, in addition to possible algebraic singularities at the endpoints $x= \pm 1$. To the best of our knowledge, the expansion we derive in this work has not been given before. (The case of algebraic-logarithmic singularities seems to be more complex, and we propose to treat this case in a future publication.) For earlier related work, see the papers by Jain and Chawla [5] and by Cîrulis [4].

The plan of this paper is as follows: In the next section, we describe the functions $f(x)$ we wish to treat in the sequel. In Section 3, we recall three theorems that are relevant to our problem: (i) a theorem of 12 concerning the asymptotic expansion of $e_{n}[f]$ when $f(x)$ has only algebraic endpoint singularities, (ii) a theorem concerning the asymptotic expansion of $P_{n}(x)$ as $n \rightarrow \infty$, where $-1<x<1$, and (iii) a theorem concerning the asymptotic expansion of integrals of the form $\int_{\alpha}^{\beta} h(\theta) e^{ \pm \mathrm{i} \nu \theta} d \theta$ as $\nu \rightarrow \infty$, where $h(\theta)$ has only algebraic endpoint singularities. In Section 4, we state the main results of this work, in which we present (i) the full asymptotic expansion as $n \rightarrow \infty$ of integrals of the form $\int_{c}^{d} f(x) P_{n}(x) d x$, where $[c, d] \in(-1,1)$ and (a) $f \in C^{\infty}[c, d]$ or (b) $f \in C^{\infty}(c, d)$ but may have arbitrary algebraic singularities at $x=c$ and/or $x=d$, and (ii) the full asymptotic expansion as $n \rightarrow \infty$ of $e_{n}[f]$ when $f(x)$ is as described in Section 2,

One important feature of the asymptotic expansion of $e_{n}[f]$ we derive here is that it is determined exclusively by the asymptotic expansions of $f(x)$ at its points of singularity, the behavior of $f(x)$ at its points of regularity being irrelevant. As for the asymptotic expansion of $\int_{c}^{d} f(x) P_{n}(x) d x$, where $[c, d] \in(-1,1)$, it is determined only by the behavior of $f(x)$ at $x=c$ and $x=d$, whether $f(x)$ is singular or regular at these points.

The results of this work, in addition to being of interest by themselves, can have applications in asymptotic analyses involving Legendre expansions, such as integral equations, numerical quadrature, and in series of spherical harmonics.

Now, it is a well known fact that when $f(x)$ has singularities on $[-1,1]$, its Legendre series $\sum_{n=0}^{\infty} e_{n}[f] P_{n}(x)$ converges slowly; the stronger the singularities, the slower the convergence. The convergence can be accelerated by applying suitable extrapolation methods to the sequence of partial sums $S_{n}(x)=\sum_{k=0}^{n} e_{k}[f] P_{k}(x)$, $n=0,1, \ldots$, of the series. In order to be able to make an educated decision as to which extrapolation method to use, and how to tune it properly, it is important that we have some qualitative information about the asymptotic expansion of 
$S_{n}[f]$ as $n \rightarrow \infty$. This information can be deduced rigorously from the asymptotic expansion of $e_{n}[f]$ as $n \rightarrow \infty$. It is not necessary to know the exact asymptotic expansion of $e_{n}[f]$ to make this deduction; knowledge of the form of this asymptotic expansion suffices. In Section 6, we discuss the consequences of this in relation to convergence acceleration. For a detailed treatment of this topic with applications, see Sidi [10], [11, Chapters 6, 12, 13], 13], for example.

\section{Assumptions on $f(x)$}

It is known (see Olver [7, p. 129], for example) that the Legendre polynomial $P_{n}(x)$ behaves like a trigonometric function for $-1<x<1$, namely, as in

$$
P_{n}(\cos \theta)=\left(\frac{2}{\pi n \sin \theta}\right)^{1 / 2} \sin \left(n \theta+\frac{\theta}{2}+\frac{\pi}{4}\right)+O\left(n^{-3 / 2}\right) \quad \text { as } n \rightarrow \infty .
$$

In addition, there are known results on the asymptotic behavior of Fourier integrals $\int_{\alpha}^{\beta} h(\theta) e^{ \pm \mathrm{i} \nu \theta} d \theta$ as $\nu \rightarrow \infty$, where $(\alpha, \beta)$ is a finite interval and $h \in C^{\infty}(\alpha, \beta)$, with possible singularities at $\alpha$ and/or $\beta$. Thus, it seems that one can exploit these facts in the asymptotic analysis of $e_{n}[f]$ as $n \rightarrow \infty$. Indeed, this turns out to be the case, provided we make the variable transformation $x=\cos \theta$ in (1.2), and re-express $e_{n}[f]$ as an integral over $\theta$ as follows:

$$
e_{n}[f]=(n+1 / 2) \int_{0}^{\pi} F(\theta) P_{n}(\cos \theta) d \theta, \quad F(\theta)=\sin \theta f(\cos \theta) .
$$

This representation of $e_{n}[f]$ suggests that we should look at the singularity structure of $F(\theta)$ for $0 \leq \theta \leq \pi$. Clearly, as $\sin \theta$ is an entire function, the singularity structure of $F(\theta)$ is determined only by that of $f(x)$.

We now state the main assumptions on the function $F(\theta)$ in (2.2):

(1) $F(\theta)$ has $m$ points of singularity in the interior of $(0, \pi)$. We denote these points by $\theta_{1}, \ldots, \theta_{m}$, and order them as in

$$
0=\theta_{0}<\theta_{1}<\theta_{2}<\cdots<\theta_{m}<\theta_{m+1}=\pi .
$$

We assume that $F(\theta)$ is infinitely differentiable at all other points of $(0, \pi)$, hence on each of the intervals $\left(\theta_{r}, \theta_{r+1}\right), r=0,1, \ldots, m$.

(2) At each of the points $\theta_{r}, F(\theta)$ has asymptotic expansions, from the right and from the left of $\theta_{r}$, that are of the form

$$
F(\theta) \sim \sum_{s=0}^{\infty} T_{r s}^{( \pm)}\left|\theta-\theta_{r}\right|^{\gamma_{r s}^{( \pm)}} \quad \text { as } \theta \rightarrow \theta_{r} \pm
$$

where $T_{r s}^{( \pm)} \neq 0$ and $\gamma_{r s}^{( \pm)}$are, in general, complex, and the $\gamma_{r s}^{( \pm)}$satisfy

$$
-1<\Re \gamma_{r 0}^{( \pm)} \leq \Re \gamma_{r 1}^{( \pm)} \leq \Re \gamma_{r 2}^{( \pm)} \leq \cdots ; \quad \lim _{s \rightarrow \infty} \Re \gamma_{r s}^{( \pm)}=+\infty
$$

Here, $\Re z$ stands for the real part of $z$. Of course, the asymptotic expansion from the left is irrelevant at $\theta_{0}=0$. Similarly, the asymptotic expansion from the right is irrelevant at $\theta_{m+1}=\pi$. Clearly, under (2.4) and (2.5), $F \in L_{1}[0, \pi]$ and $f \in L_{1}[-1,1]$.

Note also that

$$
\left|\theta-\theta_{r}\right|^{\gamma_{r s}^{(+)}}=\left(\theta-\theta_{r}\right)^{\gamma_{r s}^{(+)}}, \quad\left|\theta-\theta_{r}\right|^{\gamma_{r s}^{(-)}}=\left(\theta_{r}-\theta\right)^{\gamma_{r s}^{(-)}}
$$


For future use, we also state the asymptotic expansions of $f(x)$ at the endpoints $x=-1$ and $x=1$ [instead of those of $F(\theta)$ at $\theta=\theta_{m+1}=\pi$ and $\theta=\theta_{0}=0$, respectively], separately, as follows:

$$
f(x) \sim \sum_{s=0}^{\infty} A_{s}^{( \pm)}|x \mp 1|^{\alpha_{s}^{( \pm)}} \quad \text { as } x \rightarrow \pm 1,
$$

where $A_{s}^{( \pm)}$are nonzero constants and

$$
-1<\Re \alpha_{0}^{( \pm)}<\Re \alpha_{1}^{( \pm)}<\Re \alpha_{2}^{( \pm)}<\cdots ; \quad \lim _{s \rightarrow \infty} \Re \alpha_{s}^{( \pm)}=0 .
$$

As will be clear from Remark 2 below, the asymptotic expansion of $F(\theta)$ as $\theta \rightarrow \theta_{m+1}=\pi$ and $\theta \rightarrow \theta_{0}=0$ given in (2.4) are actually implied by those of $f(x)$ as $x \rightarrow-1$ and $x \rightarrow 1$, respectively, given in (2.7), and vice versa.

(3) By (2.4), we mean that, for each $p=0,1, \ldots$,

$$
F(\theta)-\sum_{s=0}^{p-1} T_{r s}^{( \pm)}\left|\theta-\theta_{r}\right|^{\gamma_{r s}^{( \pm)}}=O\left(\left|\theta-\theta_{r}\right|^{\gamma_{r p}^{( \pm)}}\right) \quad \text { as } \theta \rightarrow \theta_{r} \pm .
$$

(4) The asymptotic expansions in (2.4) are termwise differentiable an infinite number of times. That is, for each $k=1,2, \ldots$, the $k$ th derivative of $F(\theta)$ also has asymptotic expansions as $\theta \rightarrow \theta_{r} \pm$ that are obtained by differentiating those in (2.4) term by term.

(5) Following (2.4), we have stated that $T_{r s}^{( \pm)} \neq 0$. This is necessarily the right statement when the series $\sum_{s=0}^{\infty} T_{r s}^{( \pm)}\left|\theta-\theta_{r}\right|_{r s}^{( \pm)}$contains infinitely many terms. When it contains finitely many terms, we write $T_{r s}^{( \pm)} \neq 0$ to mean that the terms in this finite summation are all nonzero. (In any case, $T_{r 0}^{( \pm)} \neq 0$.) We adopt this convention in the remainder of this work with other asymptotic expansions, such as those in (2.7), (2.10), (3.1), and (3.9).

Remarks.

(1) The following are consequences of (2.5):

(i) For each $r$, there are only a finite number of $\gamma_{r s}^{(+)}$that have the same real parts. Similarly, for each $r$, there are only a finite number of $\gamma_{r s}^{(-)}$ that have the same real parts. Consequently, $\Re \gamma_{r s}^{(+)}<\Re \gamma_{r, s+1}^{(+)}$and $\Re \gamma_{r s^{\prime}}^{(-)}<\Re \gamma_{r, s^{\prime}+1}^{(-)}$for infinitely many values of the indices $s$ and $s^{\prime}$.

(ii) The sequences $\left\{\left|\theta-\theta_{r}\right|_{r s}^{( \pm)}\right\}_{s=0}^{\infty}$ are asymptotic scales as $\theta \rightarrow \theta_{r} \pm$, in the following sense:

$$
\lim _{\theta \rightarrow \theta_{r} \pm} \frac{\left|\theta-\theta_{r}\right|^{\gamma_{r, s+1}^{( \pm)}}}{\left|\theta-\theta_{r}\right|^{\gamma_{r s}^{( \pm)}}}= \begin{cases}0 & \text { if } \Re \gamma_{r s}^{( \pm)}<\Re \gamma_{r, s+1}^{( \pm)}, \\ 1 & \text { if } \Re \gamma_{r s}^{( \pm)}=\Re \gamma_{r, s+1}^{( \pm)}\end{cases}
$$

These limits are zero an infinite number of times since $\Re \gamma_{r s}^{(+)}<\Re \gamma_{r, s+1}^{(+)}$ and $\Re \gamma_{r s^{\prime}}^{(-)}<\Re \gamma_{r, s^{\prime}+1}^{(-)}$for infinitely many integers $s$ and $s^{\prime}$. (For a discussion of asymptotic scales, see Olver [7, p. 25], for example.) In view of (2.5) and (2.9), the expansions in (2.4) are thus genuine asymptotic expansions. 
(2) The singularity structure of $F(\theta)$ on $[0, \pi]$ is actually of the same nature as that of $f(x)$ on $[-1,1]$. To see this, assume that $f(x)$ is singular at $x=c \in(-1,1)$ with

$$
f(x) \sim \sum_{s=0}^{\infty} W_{s}^{( \pm)}|x-c|^{\delta_{s}^{( \pm)}} \quad \text { as } x \rightarrow c \pm,
$$

where $W_{s}^{( \pm)}$are nonzero constants and

$$
-1<\Re \delta_{0}^{( \pm)} \leq \Re \delta_{1}^{( \pm)} \leq \Re \delta_{2}^{( \pm)} \cdots ; \quad \lim _{s \rightarrow \infty} \Re \delta_{s}^{( \pm)}=\infty .
$$

Then, letting $\xi=\cos ^{-1} c$, and realizing that $x \rightarrow c \pm$ if and only if $\theta \rightarrow \xi \mp$, we have

$$
F(\theta) \sim \sin \theta \sum_{s=0}^{\infty} W_{s}^{( \pm)}|\cos \theta-\cos \xi|^{\delta_{s}^{( \pm)}} \quad \text { as } \theta \rightarrow \xi \mp .
$$

Re-expanding about $\theta=\xi$ [by expanding $\sin \theta$ and $(\cos \theta-\cos \xi)$ about $\theta=\xi]$, we obtain

$$
F(\theta) \sim \sum_{s=0}^{\infty} \sum_{i=0}^{\infty} \widetilde{W}_{s i}^{( \pm)}|\theta-\xi|^{\delta_{s}^{( \pm)}+i} \quad \text { as } \theta \rightarrow \xi \mp ; \quad \widetilde{W}_{00}^{( \pm)}=W_{0}^{( \pm)}(\sin \xi)^{1+\delta_{0}^{( \pm)}} .
$$

By ordering the $\left(\delta_{s}^{( \pm)}+i\right)$ according to the size of their real parts, and renaming the distinct ones by $\gamma_{s}^{(\mp)}$, we obtain an expansion for $F(\theta)$ as $\theta \rightarrow \xi \mp$ of the form

$$
F(\theta) \sim \sum_{s=0}^{\infty} \widehat{T}_{s}^{( \pm)}|\theta-\xi|^{\gamma_{s}^{( \pm)}} \quad \text { as } \theta \rightarrow \xi \pm,
$$

with

$\widehat{T}_{0}^{( \pm)}=\widetilde{W}_{00}^{(\mp)}=W_{0}^{(\mp)}(\sin \xi)^{1+\delta_{0}^{(\mp)}}$ and $\gamma_{0}^{( \pm)}=\delta_{0}^{(\mp)}$ when $\Re \delta_{0}^{( \pm)}<\Re \delta_{1}^{( \pm)}$.

This expansion is exactly of the form given in (2.4) and (2.5).

In view of this and the assumptions we have made about $F(\theta)$, we also see that, to each point of singularity $x_{r}$ of $f(x)$, there corresponds a unique point of singularity $\theta_{r}$ of $F(\theta)$, and vice versa. Between two consecutive points of singularity, the two functions are infinitely differentiable simultaneously.

As an instructive example, let us consider the case in which $f(x)$ has a finite jump discontinuity at $c \in(-1,1)$, but is infinitely differentiable in sufficiently small right and left neighborhoods of $c$. In this case, we have

$$
f(x) \sim \sum_{s=0}^{\infty} \frac{f^{(s)}(c \pm)}{s !}(x-c)^{s} \quad \text { as } x \rightarrow c \pm .
$$

Note that these are simply the two Taylor series of $f(x)$ that are valid for $x>c$ and $x<c$. Assuming that these Taylor series are full, it is clear that $f(x)$ satisfies (2.10) and (2.11) with

$$
W_{s}^{(+)}=\frac{f^{(s)}(c+)}{s !}, \quad W_{s}^{(-)}=(-1)^{s} \frac{f^{(s)}(c-)}{s !}, \quad \text { and } \quad \delta_{s}^{( \pm)}=s .
$$


As a result, $F(\theta)$ has asymptotic expansions of the form given in (2.12) and (2.13), with $\gamma_{s}^{( \pm)}=s$ for all $s$. Thus, $F(\theta)$ has a finite jump discontinuity at $\xi$.

Clearly, this treatment also covers the cases in which $c= \pm 1$.

(3) As for the fourth assumption on the termwise differentiability of the asymptotic expansions in (2.4), we mention that this assumption is crucial. It is automatically satisfied if the asymptotic expansions of $f(x)$ at its singular points can be differentiated termwise; this occurs in many cases of practical interest. One such example is that for which $f(x)$ has endpoint singularities and is of the form $f(x)=(1-x)^{\alpha}(1+x)^{\beta} g(x)$, where $g \in C^{\infty}[-1,1]$. For this case, we have

$$
\begin{gathered}
f(x) \sim \sum_{s=0}^{\infty}(-1)^{s} \frac{g_{-}^{(s)}(1)}{s !}|x-1|^{\alpha+s} \quad \text { as } x \rightarrow 1-, \quad g_{-}(x)=(1+x)^{\beta} g(x), \\
f(x) \sim \sum_{s=0}^{\infty} \frac{g_{+}^{(s)}(-1)}{s !}|x+1|^{\beta+s} \quad \text { as } x \rightarrow-1+, \quad g_{+}(x)=(1-x)^{\alpha} g(x) .
\end{gathered}
$$

Note that the first expansion is nothing but the product of $(1-x)^{\alpha}$ and the Taylor series of $g_{-}(x)$ at $x=1$, while the second expansion is the product of $(1+x)^{\beta}$ and the Taylor series of $g_{+}(x)$ at $x=-1$. By the fact that $f^{(k)} \in C^{\infty}(-1,1)$ for all $k \geq 0$, it is easy to show for each $k=1,2, \ldots$, that $f^{(k)}(x)$, just like $f(x)$, has asymptotic expansions as $x \rightarrow 1-$ and as $x \rightarrow-1+$, and that termwise differentiation $k$ times of the asymptotic expansions of $f(x)$ results in the same expansions.

It is easy to see that the same arguments apply to interior singularities.

\section{REVIEW OF NECESSARY BACKGROUND}

To derive the asymptotic expansions mentioned in Section 1, we will need three known theorems, and we state them in this section for future reference and use. The first concerns the asymptotic expansion as $n \rightarrow \infty$ of $e_{n}[f]$ when $f(x)$ has no singularities in $(-1,1)$, but may have general algebraic singularities at one or both endpoints $x= \pm 1$. The second concerns the full asymptotic expansion as $n \rightarrow \infty$ of $P_{n}(x)$ for $-1<x<1$. The third concerns the asymptotic expansion as $\nu \rightarrow \infty$ of Fourier integrals of the form $\int_{\alpha}^{\beta} h(\theta) e^{ \pm \mathrm{i} \nu \theta} d \theta$ when $h \in C^{\infty}(\alpha, \beta)$, but may have general algebraic singularities at $\theta=\alpha$ and/or $\theta=\beta$.

3.1. Asymptotic expansion of $e_{n}[f]$ when $f(x)$ has no interior singularities. As mentioned earlier, the case when $f(x)$ has no interior singularities in $(-1,1)$ [that is, when $m=0$ in [2.3)] is considered in Sidi [12. For future use and reference, we summarize the main result of [12] that concerns purely algebraic endpoint singularities and that is relevant to us here. 
Theorem 3.1. Let $f(x)$ be exactly as described above, with $m=0$ [that is, $f \in$ $C^{\infty}(-1,1)$, hence has no interior singularities]. Let

$$
\begin{aligned}
& f(x) \sim \sum_{s=0}^{\infty} A_{s}(1-x)^{\alpha_{s}} \quad \text { as } x \rightarrow 1- \\
& f(x) \sim \sum_{s=0}^{\infty} B_{s}(1+x)^{\beta_{s}} \quad \text { as } x \rightarrow-1+
\end{aligned}
$$

where

$$
\begin{aligned}
& -1<\Re \alpha_{0} \leq \Re \alpha_{1} \leq \Re \alpha_{2} \leq \cdots ; \lim _{s \rightarrow \infty} \Re \alpha_{s}=\infty, \\
& -1<\Re \beta_{0} \leq \Re \beta_{1} \leq \Re \beta_{2} \leq \cdots ; \lim _{s \rightarrow \infty} \Re \beta_{s}=\infty,
\end{aligned}
$$

and $A_{s}$ and $B_{s}$ are nonzero constants. Assume also that the asymptotic expansions in (3.1) are termwise differentiable an infinite number of times. Then, with $\widehat{n}=$ $n+1 / 2$ and $\mathbb{Z}^{+}=\{0,1,2, \ldots\}, e_{n}[f]$ has the asymptotic expansion

$$
\begin{aligned}
e_{n}[f] \sim & \sum_{\substack{s=0 \\
\alpha_{s} \notin \mathbb{Z}^{+}}}^{\infty} A_{s} \sum_{k=0}^{\infty} \frac{c_{k}\left(\alpha_{s}\right)}{\widehat{\widehat{n}}^{2\left(\alpha_{s}+k+1 / 2\right)}} \\
& +(-1)^{n} \sum_{\substack{s=0 \\
\beta_{s} \notin \mathbb{Z}^{+}}}^{\infty} B_{s} \sum_{k=0}^{\infty} \frac{c_{k}\left(\beta_{s}\right)}{\widehat{n}^{2\left(\beta_{s}+k+1 / 2\right)}} \text { as } n \rightarrow \infty .
\end{aligned}
$$

Here, $c_{k}(\omega)$ are given as in

$$
c_{k}(\omega)=2^{\omega+1} \frac{\Gamma(1+\omega)}{\Gamma(-\omega)} \frac{B_{2 k}^{(\sigma)}(\sigma / 2)}{(2 k) !} \frac{\Gamma(2 k+2 \omega+2)}{\Gamma(2 \omega+2)}, \quad k=0,1, \ldots ; \quad \sigma=-2 \omega-1,
$$

and are analytic functions of $\omega$ for $\Re \omega>-1 . B_{s}^{(\sigma)}(u)$ is the sth generalized Bernoulli polynomial 1 When $\omega \in \mathbb{Z}^{+}$, there holds $c_{k}(\omega)=0$ for each $k=0,1, \ldots$.

Remarks.

(1) An interesting feature of the asymptotic expansion of $e_{n}[f]$ given in (3.3) is that it can be written down easily by looking only at the asymptotic expansions of $f(x)$ as $x \rightarrow \pm 1$, which are given in (3.1), nothing else being needed.

(2) Another feature of this expansion is that the powers $(1 \mp x)^{s}, s \geq 0$ integer, even when they are present in the asymptotic expansions of $f(x)$ as $x \rightarrow \pm 1$ given in (3.1), do not contribute to the asymptotic expansion of $e_{n}[f]$. Thus, if $f(x)$ is a regular function, then $\alpha_{s}$ and $\beta_{s}$ in (3.1) are all nonnegative integers; consequently, the summations in (3.3) are both empty, and this means that $e_{n}[f]=O\left(n^{-\mu}\right)$ as $n \rightarrow \infty$ for every $\mu>0$.

\footnotetext{
1 The generalized Bernoulli polynomials $B_{s}^{(\sigma)}(u)$ are defined via (see Andrews, Askey, and Roy [2. p. 615], for example)

$$
\left(\frac{t}{e^{t}-1}\right)^{\sigma} e^{u t}=\sum_{s=0}^{\infty} B_{s}^{(\sigma)}(u) \frac{t^{s}}{s !}, \quad|t|<2 \pi .
$$

They satisfy $B_{s}^{(\sigma)}(\sigma-u)=(-1)^{s} B_{s}^{(\sigma)}(u)$; hence $B_{s}^{(\sigma)}(\sigma / 2)=0$ for $s=1,3,5, \ldots . B_{s}^{(\sigma)}(0)$ are called the generalized Bernoulli numbers and are denotedR by $B_{s}^{(\sigma)}$. Note that $B_{0}^{(\sigma)}=1$ for all $\sigma$. In addition, $B_{k}^{(\sigma)}(u)=\sum_{s=0}^{k}\left(\begin{array}{l}k \\ s\end{array}\right) B_{k-s}^{(\sigma)} u^{s}$ for all $k$.
} 
3.2. Asymptotic expansion of $P_{n}(x)$ when $-1<x<1$. Asymptotic expansions for $P_{n}(x)$ with $-1<x<1$ come in different forms in the literature. See Olver [7, for example. There is one particular form that is suitable for us, and this is given in Szegö [15, p. 196, Theorem 8.21.9]. A slightly modified version of this theorem, restricted to our problem, is as follows:

Theorem 3.2. There exist analytic functions $\phi_{k}(z)$ that are regular for $|z|=1$, $z \neq \pm 1$, such that, with $\widehat{n}=n+1 / 2$,

$$
P_{n}(\cos \theta) \sim \Re\left\{e^{\mathrm{i} \widehat{ } \hat{n} \theta} \sum_{k=0}^{\infty} \frac{\phi_{k}\left(e^{\mathrm{i} \theta}\right)}{\widehat{n}^{k+1 / 2}}\right\} \text { as } n \rightarrow \infty,
$$

uniformly for $\epsilon \leq \theta \leq \pi-\epsilon$, with $\epsilon \in(0, \pi / 4)$ fixed. That is, for each $p=0,1, \ldots$, and for $0<\theta<\pi$, there holds

$$
P_{n}(\cos \theta)=\Re\left\{e^{\mathrm{i} \widehat{ } \hat{n} \theta}\left[\sum_{k=0}^{p-1} \frac{\phi_{k}\left(e^{\mathrm{i} \theta}\right)}{\widehat{n}^{k+1 / 2}}+R_{p, n}(\theta)\right]\right\},
$$

where

$$
R_{p, n}(\theta)=O\left(\widehat{n}^{-p-1 / 2}\right) \quad \text { as } n \rightarrow \infty, \text { uniformly for } \epsilon \leq \theta \leq \pi-\epsilon .
$$

Actually, with $D_{\theta}=d / d \theta$,

$$
\phi_{k}\left(e^{\mathrm{i} \theta}\right)=(-1)^{k} \frac{2}{\pi^{1 / 2}}\left(\begin{array}{c}
-1 / 2 \\
k
\end{array}\right) e^{\mathrm{i}(\theta-\pi) / 2} B_{k}^{(1 / 2)}\left((\mathrm{i} / 2) D_{\theta}\right)\left[\left(1-e^{\mathrm{i} 2 \theta}\right)^{-1 / 2}\right] .
$$

Here, $B_{k}^{(\sigma)}(y)=\sum_{s=0}^{k}\left(\begin{array}{l}k \\ s\end{array}\right) B_{k-s}^{(\sigma)} y^{s}$ is the $k$ th generalized Bernoulli polynomial (see footnote 1), and, by $B_{k}^{(\sigma)}\left(c D_{\theta}\right) Y(\theta)$, we mean

$$
B_{k}^{(\sigma)}\left(c D_{\theta}\right) Y(\theta)=\sum_{s=0}^{k}\left(\begin{array}{l}
k \\
s
\end{array}\right) B_{k-s}^{(\sigma)} c^{s}\left[D_{\theta}^{s} Y(\theta)\right]=\sum_{s=0}^{k}\left(\begin{array}{l}
k \\
s
\end{array}\right) B_{k-s}^{(\sigma)} c^{s} Y^{(s)}(\theta) .
$$

Remark. In Theorem A.1 of the appendix to this work, we derive the full asymptotic expansions of $P_{\nu}^{\mu}(x)$ and $Q_{\nu}^{\mu}(x)$, the associated Legendre functions of the first and second kinds, respectively, when $\nu$ and $\mu$ are real and $\mu<1 / 2$. Theorem 3.2 follows from Theorem A.1, it can be obtained by setting $\mu=0, \nu=n$, and $\widehat{\nu}=\widehat{n}=n+1 / 2$ in Theorem A.1 and recalling that $P_{n}^{0}(x)=P_{n}(x)$. We also note that explicit expressions for the $\phi_{k}(z)$ are not given in [15].

3.3. Asymptotic expansion of $\int_{\alpha}^{\beta} h(\theta) e^{ \pm \mathrm{i} \nu \theta} d \theta$. We also need a result on asymptotic expansions of finite-range Fourier integrals of functions with algebraic singularities. The following theorem is actually a special case of one given in Bleistein and Handelsman [3, Sections 3.4, 6.3, and 6.4]. For a simple proof, see Sidi [14]:

Theorem 3.3. Let $\alpha<\beta$, and let $h \in C^{\infty}(\alpha, \beta)$ and satisfy

$$
\begin{aligned}
& h(\theta) \sim \sum_{s=0}^{\infty} A_{s}(\theta-\alpha)^{\rho_{s}} \quad \text { as } \theta \rightarrow \alpha+, \\
& h(\theta) \sim \sum_{s=0}^{\infty} B_{s}(\beta-\theta)^{\sigma_{s}} \quad \text { as } \theta \rightarrow \beta-,
\end{aligned}
$$


such that $A_{s}$ and $B_{s}$ are nonzero constants, and

$$
\begin{aligned}
& -1<\Re \rho_{0} \leq \Re \rho_{1} \leq \Re \rho_{2} \leq \cdots ; \lim _{s \rightarrow \infty} \Re \rho_{s}=\infty, \\
& -1<\Re \sigma_{0} \leq \Re \sigma_{1} \leq \Re \sigma_{2} \leq \cdots ; \lim _{s \rightarrow \infty} \Re \sigma_{s}=\infty .
\end{aligned}
$$

Assume also that the asymptotic expansions in (3.9) are termwise differentiable an infinite number of times. Then

$$
\int_{\alpha}^{\beta} h(\theta) e^{ \pm \mathrm{i} \nu \theta} d \theta \sim e^{ \pm \mathrm{i} \nu \alpha} \sum_{s=0}^{\infty} A_{s} \frac{\Gamma\left(\rho_{s}+1\right)}{(\mp \mathrm{i} \nu)^{\rho_{s}+1}}+e^{ \pm \mathrm{i} \nu \beta} \sum_{s=0}^{\infty} B_{s} \frac{\Gamma\left(\sigma_{s}+1\right)}{( \pm \mathrm{i} \nu)^{\sigma_{s}+1}} \quad \text { as } \nu \rightarrow \infty .
$$

Remarks.

(1) It is important to note that, once it is established that $h(\theta)$ is infinitely differentiable in the open interval $(\alpha, \beta)$, the asymptotic expansion of $\int_{\alpha}^{\beta} h(\theta) e^{ \pm \mathrm{i} \nu \theta} d \theta$ is determined by the asymptotic expansions of $h(\theta)$ at the endpoints of $(\alpha, \beta)$.

(2) In case $f \in C^{\infty}(\alpha, \beta)$ and has a full Taylor series at $\theta=\alpha$ and/or at $\theta=\beta$, we have $A_{s}=h^{(s)}(\alpha) / s$ ! and/or $B_{s}=(-1)^{s} h^{(s)}(\beta) / s$ !, and $\rho_{s}=\sigma_{s}=s$, $s=0,1, \ldots$.

(3) In addition, if $h(\theta)$ is infinitely differentiable at $\alpha$ such that $h^{(i)}(\alpha)=0$ for all $i \geq 0$ [or at $\beta$ such that $h^{(i)}(\beta)=0$ for all $i \geq 0$ ], then the asymptotic expansion of $h(\theta)$ as $\theta \rightarrow \alpha$ (or as $\theta \rightarrow \beta$ ) is empty or, equivalently, it is zero. In such a case, the endpoint $\alpha$ (or $\beta$ ) has no contribution to the asymptotic expansion of $\int_{\alpha}^{\beta} h(\theta) e^{ \pm \mathrm{i} \nu \theta} d \theta$ as $\nu \rightarrow \infty$.

(4) In case $h^{(i)}(\alpha)=h^{(i)}(\beta)=0$ for all $i \geq 0$, the asymptotic expansion of $I(\nu)=\int_{\alpha}^{\beta} h(\theta) e^{ \pm \mathrm{i} \nu \theta} d \theta$ as $\nu \rightarrow \infty$ is empty, hence zero. Of course, this does not mean that $I(\nu)$ is zero. However, it does mean that $I(\nu)$ tends to zero as $\nu \rightarrow \infty$ faster than any negative power of $\nu$; that is, $I(\nu)=O\left(\nu^{-\tau}\right)$ as $\nu \rightarrow \infty$ for every $\tau>0$.

(5) Finally, the asymptotic expansions in 3.11 are valid also when $\Re \alpha_{s} \leq$ -1 and/or $\Re \beta_{s} \leq-1$ as long as $\alpha_{s}, \beta_{s} \neq-1,-2, \ldots$. In such cases,

the integrals $\int_{\alpha}^{\beta} h(\theta) e^{ \pm \mathrm{i} \nu \theta} d \theta$ diverge in the ordinary sense but do exist as Hadamard finite parts. For this point, see [14].

\section{MAin RESUlts}

4.1. Asymptotic expansion of $\int_{c}^{d} f(x) P_{n}(x) d x,-1<c<d<1$.

Theorem 4.1. Let $-1<c<d<1$, and assume that $f \in C^{\infty}(c, d)$, which implies that $F(\theta)=\sin \theta f(\cos \theta)$ is in $C^{\infty}(\alpha, \beta)$, where $\alpha=\cos ^{-1} d$ and $\beta=\cos ^{-1} c$ and $0<\alpha<\beta<\pi$. Assume that, as $x \rightarrow c$ and as $x \rightarrow d, f(x)$ is such that $F(\theta)$ has the asymptotic expansions (cf. Remark 2 in Section 2 )

$$
\begin{aligned}
& F(\theta) \sim \sum_{s=0}^{\infty} U_{s}(\theta-\alpha)^{\rho_{s}} \quad \text { as } \theta \rightarrow \alpha+; \quad \alpha=\cos ^{-1} d>0, \\
& F(\theta) \sim \sum_{s=0}^{\infty} V_{s}(\beta-\theta)^{\sigma_{s}} \quad \text { as } \theta \rightarrow \beta-; \quad \beta=\cos ^{-1} c<\pi,
\end{aligned}
$$


where

$$
\begin{aligned}
& -1<\Re \rho_{0} \leq \Re \rho_{1} \leq \Re \rho_{2} \leq \cdots ; \lim _{s \rightarrow \infty} \Re \rho_{s}=\infty, \\
& -1<\Re \sigma_{0} \leq \Re \sigma_{1} \leq \Re \sigma_{2} \leq \cdots ; \lim _{s \rightarrow \infty} \Re \sigma_{s}=\infty,
\end{aligned}
$$

and $U_{s}$ and $V_{s}$ are nonzero constants. Assume also that these asymptotic expansions can be differentiated termwise an infinite number of times. With the functions $\phi_{k}(z)$ as in Theorem 3.2 , for arbitrary $\theta \in[\alpha, \beta]$, let

$$
\phi_{k j}(\theta)=\frac{1}{j !} \frac{d^{j}}{d \theta^{j}} \phi_{k}\left(e^{\mathrm{i} \theta}\right), \quad j, k=0,1, \ldots,
$$

and

$$
\begin{aligned}
& G_{\mu}^{(+)}(\theta ; \omega)=\frac{1}{2} \sum_{\substack{j, k \geq 0 \\
j+k=\mu}} \mathrm{i}^{\omega+j+1} \phi_{k j}(\theta) \Gamma(\omega+j+1), \\
& \widehat{G}_{\mu}^{(+)}(\theta ; \omega)=\frac{1}{2} \sum_{\substack{j, k \geq 0 \\
j+k=\mu}}(-\mathrm{i})^{\omega+j+1} \overline{\phi_{k j}(\theta)} \Gamma(\omega+j+1), \\
& G_{\mu}^{(-)}(\theta ; \omega)=\frac{1}{2} \sum_{\substack{j, k \geq 0 \\
j+k=\mu}}(-1)^{j}(-\mathrm{i})^{\omega+j+1} \phi_{k j}(\theta) \Gamma(\omega+j+1), \\
& \widehat{G}_{\mu}^{(-)}(\theta ; \omega)=\frac{1}{2} \sum_{\substack{j, k \geq 0 \\
j+k=\mu}}(-1)^{j} \mathrm{i}^{\omega+j+1} \overline{\phi_{k j}(\theta)} \Gamma(\omega+j+1) .
\end{aligned}
$$

Then, as $n \rightarrow \infty$,

$$
\begin{aligned}
\int_{c}^{d} f(x) P_{n}(x) d x & \sim e^{\mathrm{i} \widehat{n} \alpha} \sum_{s=0}^{\infty} U_{s} \sum_{\mu=0}^{\infty} \frac{G_{\mu}^{(+)}\left(\alpha ; \rho_{s}\right)}{\widehat{n}^{\rho_{s}+\mu+3 / 2}}+e^{-\mathrm{i} \widehat{ } \alpha} \sum_{s=0}^{\infty} U_{s} \sum_{\mu=0}^{\infty} \frac{\widehat{G}_{\mu}^{(+)}\left(\alpha ; \rho_{s}\right)}{\widehat{n}^{\rho_{s}+\mu+3 / 2}} \\
& +e^{\mathrm{i} \widehat{n} \beta} \sum_{s=0}^{\infty} V_{s} \sum_{\mu=0}^{\infty} \frac{G_{\mu}^{(-)}\left(\beta ; \sigma_{s}\right)}{\widehat{n}^{\sigma_{s}+\mu+3 / 2}}+e^{-\mathrm{i} \widehat{\mathrm{n}} \beta} \sum_{s=0}^{\infty} V_{s} \sum_{\mu=0}^{\infty} \frac{\widehat{G}_{\mu}^{(-)}\left(\beta ; \sigma_{s}\right)}{\widehat{n}^{\sigma_{s}+\mu+3 / 2}} .
\end{aligned}
$$

Remarks.

(1) The case $f \in C^{\infty}[c, d]$ is actually included in Theorem 4.1, In this case, $F \in C^{\infty}[\alpha, \beta]$ necessarily. Therefore, when $F(\theta)$ has full Taylor series at $\alpha$ and $\beta$, (4.1) holds with

$U_{s}=\frac{F^{(s)}(\alpha)}{s !}, \quad \rho_{s}=s \quad$ and $\quad V_{s}=(-1)^{s} \frac{F^{(s)}(\beta)}{s !}, \quad \sigma_{s}=s, \quad s=0,1, \ldots$.

(2) Note that the only contributions to the asymptotic expansion in (4.5) come from the endpoints $x=c$ and $x=d$. In addition, it is quite easy to write down these contributions; we simply replace $(\theta-\alpha)^{\rho_{s}}$ and $(\beta-\theta)^{\sigma_{s}}$ in (4.1) by appropriate asymptotic expansions in terms of the powers $\left\{\widehat{n}^{-\left(\rho_{s}+\mu+3 / 2\right)}\right\}_{\mu=0}^{\infty}$ and $\left\{\widehat{n}^{-\left(\sigma_{s}+\mu+3 / 2\right)}\right\}_{\mu=0}^{\infty}$, respectively, with coefficients that are independent of the $U_{s}$ and $V_{s}$.

(3) It is easy to see that, when $\omega$ is real, there holds

$$
\widehat{G}_{\mu}^{( \pm)}(\theta ; \omega)=\overline{G_{\mu}^{( \pm)}(\theta ; \omega)} \text {. }
$$


Therefore, if the $U_{s}, V_{s}, \rho_{s}$, and $\sigma_{s}$ are all real, then (4.5) becomes

$$
\begin{aligned}
\int_{c}^{d} f(x) P_{n}(x) d x \sim 2 \Re & \left\{e^{\mathrm{i} \widehat{n} \alpha} \sum_{s=0}^{\infty} U_{s} \sum_{\mu=0}^{\infty} \frac{G_{\mu}^{(+)}\left(\alpha ; \rho_{s}\right)}{\widehat{n}^{\rho_{s}+\mu+3 / 2}}\right. \\
& \left.+e^{\mathrm{i} \widehat{n} \beta} \sum_{s=0}^{\infty} V_{s} \sum_{\mu=0}^{\infty} \frac{G_{\mu}^{(-)}\left(\beta ; \sigma_{s}\right)}{\widehat{n}^{\sigma_{s}+\mu+3 / 2}}\right\} .
\end{aligned}
$$

\subsection{Asymptotic expansion of $e_{n}[f]$.}

Theorem 4.2. Let $f(x)$ be such that $F(\theta)$ is precisely as described in Section 2 with the notation therein. Then, as $n \rightarrow \infty, e_{n}[f]$ has the asymptotic expansion

$$
\begin{aligned}
e_{n}[f] \sim \sum_{r=1}^{m}\left\{e^{\mathrm{i} \hat{n} \theta_{r}}\left[\sum_{s=0}^{\infty} T_{r s}^{(+)} \sum_{\mu=0}^{\infty} \frac{G_{\mu}^{(+)}\left(\theta_{r} ; \gamma_{r s}^{(+)}\right)}{\widehat{n}_{r s}^{(+)}+\mu+1 / 2}+\sum_{s=0}^{\infty} T_{r s}^{(-)} \sum_{\mu=0}^{\infty} \frac{G_{\mu}^{(-)}\left(\theta_{r} ; \gamma_{r s}^{(-)}\right)}{\widehat{n}_{r s}^{(-)}+\mu+1 / 2}\right]\right. \\
\left.+e^{-\mathrm{i} \hat{n} \theta_{r}}\left[\sum_{s=0}^{\infty} T_{r s}^{(+)} \sum_{\mu=0}^{\infty} \frac{\widehat{G}_{\mu}^{(+)}\left(\theta_{r} ; \gamma_{r s}^{(+)}\right)}{\widehat{n}_{r s}^{(+)}+\mu+1 / 2}+\sum_{s=0}^{\infty} T_{r s}^{(-)} \sum_{\mu=0}^{\infty} \frac{\widehat{G}_{\mu}^{(-)}\left(\theta_{r} ; \gamma_{r s}^{(-)}\right)}{\widehat{n}_{r s}^{(-)}+\mu+1 / 2}\right]\right\} \\
+\sum_{\substack{s=0 \\
\alpha_{s}^{(+)} \notin \mathbb{Z}^{+}}}^{\infty} A_{s}^{(+)} \sum_{k=0}^{\infty} \frac{c_{k}\left(\alpha_{s}^{(+)}\right)}{\widehat{n}^{2\left(\alpha_{s}^{(+)}+k+1 / 2\right)}}+(-1)^{n} \sum_{\substack{s=0 \\
\alpha_{s}^{(-)} \notin \mathbb{Z}^{+}}}^{\infty} A_{s}^{(-)} \sum_{k=0}^{\infty} \frac{c_{k}\left(\alpha_{s}^{(-)}\right)}{\widehat{n}^{2\left(\alpha_{s}^{(-)}+k+1 / 2\right)}}
\end{aligned}
$$

The functions $c_{k}(\omega)$ are exactly as described in Theorem 3.1. The functions $G_{\mu}^{( \pm)}(\theta ; \omega)$ and $\widehat{G}_{\mu}^{( \pm)}(\theta ; \omega)$ are as described in Theorem 4.1.

\section{Remarks.}

(1) Note that the only contributions to the asymptotic expansion in (4.8) come from the points of singularity in $[0, \pi]$. In addition, it is quite easy to write down these contributions; we simply replace $\left(\theta-\theta_{r}\right)^{\gamma_{r s}^{( \pm)}}$in (2.4) by appropriate asymptotic expansions in terms of the powers $\left\{\widehat{n}^{-\left(\gamma_{r s}^{( \pm)}+\mu+1 / 2\right)}\right\}_{\mu=0}^{\infty}$, with coefficients that are independent of the $T_{r s}^{( \pm)}$.

(2) We have chosen to express the asymptotic expansion of $e_{n}[f]$ in terms of $\widehat{n}=n+1 / 2$ instead of $n$. The reason for this is that the contributions to this asymptotic expansion coming from $x= \pm 1$ have half as many terms in powers of $\widehat{n}=n+1 / 2$ as there are in terms of powers of $n$. Of course, we can re-expand (4.8) in powers of $n$; in this case, the powers $\widehat{n}^{2\left(\alpha_{s}^{( \pm)}+k+1 / 2\right)}$ in (4.8) are replaced by $n^{2 \alpha_{s}^{( \pm)}+2 k+1}$, while the rest of the asymptotic expansions retain their forms, with $\widehat{n}$ replaced by $n$.

(3) If the $T_{r s}^{( \pm)}$and the $\gamma_{r s}^{( \pm)}$are all real, then, by (4.6), the result in (4.8) becomes

$$
\begin{gathered}
e_{n}[f] \sim 2 \Re\left\{\sum_{r=1}^{m} e^{\mathrm{i} \widehat{n} \theta_{r}}\left[\sum_{s=0}^{\infty} T_{r s}^{(+)} \sum_{\mu=0}^{\infty} \frac{G_{\mu}^{(+)}\left(\theta_{r} ; \gamma_{r s}^{(+)}\right)}{\widehat{n}_{r s}^{(+)}+\mu+1 / 2}+\sum_{s=0}^{\infty} T_{r s}^{(-)} \sum_{\mu=0}^{\infty} \frac{G_{\mu}^{(-)}\left(\theta_{r} ; \gamma_{r s}^{(-)}\right)}{\widehat{n}_{r s}^{(-)}+\mu+1 / 2}\right]\right\} \\
+\sum_{\substack{s=0 \\
\alpha_{s}^{(+)} \notin \mathbb{Z}^{+}}}^{\infty} A_{s}^{(+)} \sum_{k=0}^{\infty} \frac{c_{k}\left(\alpha_{s}^{(+)}\right)}{\widehat{n}^{2\left(\alpha_{s}^{(+)}+k+1 / 2\right)}}+(-1)^{n} \sum_{\substack{s=0 \\
\alpha_{s}^{(-)} \notin \mathbb{Z}^{+}}}^{\infty} A_{s}^{(-)} \sum_{k=0}^{\infty} \frac{c_{k}\left(\alpha_{s}^{(-)}\right)}{\widehat{n}^{2\left(\alpha_{s}^{(-)}+k+1 / 2\right)}} .
\end{gathered}
$$




\section{Proofs of main Results}

5.1. Proof of Theorem 4.1. Making the variable transformation $x=\cos \theta$, we first have

$$
\int_{c}^{d} f(x) P_{n}(x) d x=\int_{\alpha}^{\beta} \Phi_{n}(\theta) d \theta, \quad \Phi_{n}(\theta)=F(\theta) P_{n}(\cos \theta) .
$$

Following this, we define

$$
H_{k}(\theta)=F(\theta) \phi_{k}\left(e^{\mathrm{i} \theta}\right), \quad \widetilde{H}_{k}(\theta)=F(\theta) \overline{\phi_{k}\left(e^{\mathrm{i} \theta}\right)}, \quad k=0,1, \ldots,
$$

and let

$$
\Psi_{p, n}(\theta)=\frac{1}{2} \sum_{k=0}^{p-1}\left[e^{\mathrm{i} \widehat{n} \theta} H_{k}(\theta)+e^{-\mathrm{i} \widehat{n} \theta} \widetilde{H}_{k}(\theta)\right] \widehat{n}^{-k-1 / 2} .
$$

In addition, we let

$$
\widehat{\Psi}_{p, n}(\theta)=F(\theta) \Re\left[e^{\mathrm{i} \widehat{n} \theta} R_{p, n}(\theta)\right]
$$

With these, we have

$$
\Phi_{n}(\theta)=\Psi_{p, n}(\theta)+\widehat{\Psi}_{p, n}(\theta)
$$

and

$$
\int_{c}^{d} f(x) P_{n}(x) d x=\int_{\alpha}^{\beta} \Psi_{p, n}(\theta) d \theta+\int_{\alpha}^{\beta} \widehat{\Psi}_{p, n}(\theta) d \theta .
$$

We now have to derive asymptotic expansions for the integrals $\int_{\alpha}^{\beta} \Psi_{p, n}(\theta) d \theta$ and $\int_{\alpha}^{\beta} \widehat{\Psi}_{p, n}(\theta) d \theta$. By (3.7) in Theorem 3.2, it is clear that

$$
\int_{\alpha}^{\beta} \widehat{\Psi}_{p, n}(\theta) d \theta=O\left(\widehat{n}^{-p-1 / 2}\right) \quad \text { as } n \rightarrow \infty .
$$

As for $\int_{\alpha}^{\beta} \Psi_{p, n}(\theta) d \theta$, we need to analyze the asymptotic behavior of the integrals $\int_{\alpha}^{\beta} e^{\mathrm{i} \widehat{n} \theta} H_{k}(\theta) d \theta$ and $\int_{\alpha}^{\beta} e^{-\mathrm{i} \widehat{n} \theta} \widetilde{H}_{k}(\theta) d \theta$ carefully. Now, by Theorem 3.2 , the functions $\phi_{k}\left(e^{\mathrm{i} \theta}\right)$ are analytic for $\theta \in(0, \pi)$, hence have convergent Taylor series expansions about arbitrary $\eta \in(0, \pi)$, and these are given as in

$$
\phi_{k}\left(e^{\mathrm{i} \theta}\right)=\sum_{j=0}^{\infty} \phi_{k j}(\eta)(\theta-\eta)^{j} .
$$

Being Taylor series, these are also asymptotic expansions as $\theta \rightarrow \eta \pm$. By (4.1) and (5.8), we have

$$
\begin{aligned}
& H_{k}(\theta) \sim \sum_{s=0}^{\infty} U_{s} \sum_{j=0}^{\infty} \phi_{k j}(\alpha)(\theta-\alpha)^{\rho_{s}+j} \quad \text { as } \theta \rightarrow \alpha+, \\
& H_{k}(\theta) \sim \sum_{s=0}^{\infty} V_{s} \sum_{j=0}^{\infty}(-1)^{j} \phi_{k j}(\beta)(\beta-\theta)^{\sigma_{s}+j} \quad \text { as } \theta \rightarrow \beta-, \\
& \widetilde{H}_{k}(\theta) \sim \sum_{s=0}^{\infty} U_{s} \sum_{j=0}^{\infty} \overline{\phi_{k j}(\alpha)}(\theta-\alpha)^{\rho_{s}+j} \quad \text { as } \theta \rightarrow \alpha+, \\
& \widetilde{H}_{k}(\theta) \sim \sum_{s=0}^{\infty} V_{s} \sum_{j=0}^{\infty}(-1)^{j} \overline{\phi_{k j}(\beta)}(\beta-\theta)^{\sigma_{s}+j} \quad \text { as } \theta \rightarrow \beta-.
\end{aligned}
$$


Now applying Theorem 3.3, we obtain

$$
\begin{aligned}
\int_{\alpha}^{\beta} e^{\mathrm{i} \widehat{ } \hat{n} \theta} H_{k}(\theta) d \theta & \sim e^{\mathrm{i} \widehat{\hat{n}} \alpha} \sum_{s=0}^{\infty} U_{s} \sum_{j=0}^{\infty} \phi_{k j}(\alpha) \frac{\Gamma\left(\rho_{s}+j+1\right)}{(-\mathrm{i} \widehat{n})^{\rho_{s}+j+1}} \\
& +e^{\mathrm{i} \widehat{ } \widehat{n} \beta} \sum_{s=0}^{\infty} V_{s} \sum_{j=0}^{\infty}(-1)^{j} \phi_{k j}(\beta) \frac{\Gamma\left(\sigma_{s}+j+1\right)}{(\mathrm{i} \widehat{\hat{n}})^{\sigma_{s}+j+1}} \quad \text { as } n \rightarrow \infty
\end{aligned}
$$

and

$$
\begin{aligned}
& \int_{\alpha}^{\beta} e^{-\mathrm{i} \widehat{ } \hat{n} \theta} \widetilde{H}_{k}(\theta) d \theta \sim e^{-\mathrm{i} \widehat{n} \alpha} \sum_{s=0}^{\infty} U_{s} \sum_{j=0}^{\infty} \overline{\phi_{k j}(\alpha)} \frac{\Gamma\left(\rho_{s}+j+1\right)}{(\mathrm{i} \widehat{n})^{\rho_{s}+j+1}} \\
&+e^{-\mathrm{i} \widehat{ } \widehat{ } \beta} \sum_{s=0}^{\infty} V_{s} \sum_{j=0}^{\infty}(-1)^{j} \overline{\phi_{k j}(\beta)} \frac{\Gamma\left(\sigma_{s}+j+1\right)}{(-\mathrm{i} \widehat{n})^{\sigma_{s}+j+1}} \quad \text { as } n \rightarrow \infty .
\end{aligned}
$$

Substituting (5.10) and (5.11) in (5.1), rearranging, and invoking (4.4), we obtain

$$
\begin{aligned}
\int_{\alpha}^{\beta} \Psi_{p, n}(\theta) d \theta & \sim e^{\mathrm{i} \widehat{n} \alpha} \sum_{s=0}^{\infty} U_{s}\left[\sum_{\mu=0}^{p-1} \frac{G_{\mu}^{(+)}\left(\alpha ; \rho_{s}\right)}{\widehat{n}^{\rho_{s}+\mu+3 / 2}}+O\left(\widehat{n}^{-\rho_{s}-p-3 / 2}\right)\right] \\
& +e^{-\mathrm{i} \widehat{\hat{n}} \alpha} \sum_{s=0}^{\infty} U_{s}\left[\sum_{\mu=0}^{p-1} \frac{\widehat{G}_{\mu}^{(+)}\left(\alpha ; \rho_{s}\right)}{\widehat{n}^{\rho_{s}+\mu+3 / 2}}+O\left(\widehat{n}^{-\rho_{s}-p-3 / 2}\right)\right] \\
& +e^{\mathrm{i} \hat{\mathrm{n}} \beta} \sum_{s=0}^{\infty} V_{s}\left[\sum_{\mu=0}^{p-1} \frac{G_{\mu}^{(-)}\left(\beta ; \sigma_{s}\right)}{\widehat{n}^{\sigma_{s}+\mu+3 / 2}}+O\left(\widehat{n}^{-\sigma_{s}-p-3 / 2}\right)\right] \\
& +e^{-\mathrm{i} \widehat{n} \beta} \sum_{s=0}^{\infty} V_{s}\left[\sum_{\mu=0}^{p-1} \frac{\widehat{G}_{\mu}^{(-)}\left(\beta ; \sigma_{s}\right)}{\widehat{n}^{\sigma_{s}+\mu+3 / 2}}+O\left(\widehat{n}^{-\sigma_{s}-p-3 / 2}\right)\right] \quad \text { as } n \rightarrow \infty
\end{aligned}
$$

Substituting (5.7) and (5.12) in (5.6), and letting $p \rightarrow \infty$, we obtain the result in (4.5). This is a consequence of the fact that $p$ is arbitrary and that the $O$ terms in (5.7) and (5.12) all tend to zero simultaneously.

\subsection{Proof of Theorem 4.2.}

5.2.1. Introduction of neutralizers. We first introduce appropriate neutralizers that will allow us to break up the problem into two smaller subproblems.

Definition 5.1. Given $a<b$, a neutralizer $Q(x ; a, b)$ is an infinitely differentiable function on $(-\infty, \infty)$, such that, either

$$
Q(x ; a, b)= \begin{cases}0 & \text { if } x \leq a, \\ 1 & \text { if } x \geq b\end{cases}
$$

or

$$
Q(x ; a, b)= \begin{cases}1 & \text { if } x \leq a, \\ 0 & \text { if } x \geq b .\end{cases}
$$

Consequently, $Q(x ; a, b)$ also satisfies

$$
Q^{(i)}(a ; a, b)=0, \quad Q^{(i)}(b ; a, b)=0, \quad i=1,2, \ldots .
$$


Neutralizers can be constructed in different ways. For example, if we let

$$
p(x)= \begin{cases}0 & \text { if } x \leq 0 \\ e^{-1 / x} & \text { if } x>0\end{cases}
$$

then we can construct two neutralizers $Q_{+}(x ; a, b)$ and $Q_{-}(x ; a, b)$ as follows:

$$
Q_{+}(x ; a, b)=\frac{p(x-a)}{p(x-a)+p(b-x)}, \quad Q_{-}(x ; a, b)=\frac{p(b-x)}{p(x-a)+p(b-x)} .
$$

Now, $Q_{+}(x ; a, b)$ is as in (5.13), while $Q_{-}(x ; a, b)$ is as in (5.14). In addition,

$$
Q_{+}(x ; a, b)+Q_{-}(x ; a, b)=1 .
$$

Going back to our problem, let us choose $a, b, c, d$ such that

$$
0<a<b<\theta_{1}, \quad \theta_{m}<c<d<\pi .
$$

With the help of the above, we now construct two functions, $R_{+}(\theta)$ and $R_{-}(\theta)$, as follows:

$$
\begin{aligned}
& R_{+}(\theta)= \begin{cases}Q_{+}(\theta ; a, b) & \text { if } \theta<\theta_{1}, \\
1 & \text { if } \theta_{1} \leq \theta \leq \theta_{m}, \\
Q_{-}(\theta ; c, d) & \text { if } \theta>\theta_{m},\end{cases} \\
& R_{-}(\theta)= \begin{cases}Q_{-}(\theta ; a, b) & \text { if } \theta<\theta_{1}, \\
0 & \text { if } \theta_{1} \leq \theta \leq \theta_{m}, \\
Q_{+}(\theta ; c, d) & \text { if } \theta>\theta_{m} .\end{cases}
\end{aligned}
$$

Clearly, $R_{+}(\theta)$ and $R_{-}(\theta)$ are infinitely differentiable everywhere, and satisfy

$$
\begin{gathered}
R_{+}(\theta)=\left\{\begin{array}{ll}
0 & \text { if } \theta<a \text { or } \theta>d, \\
1 & \text { if } b<\theta<c,
\end{array} \quad R_{-}(x)= \begin{cases}1 & \text { if } \theta<a \text { or } \theta>d, \\
0 & \text { if } b<\theta<c,\end{cases} \right. \\
R_{+}(\theta)+R_{-}(\theta)=1,
\end{gathered}
$$

and

$$
R_{+}^{(i)}(a)=0, \quad R_{-}^{(i)}(d)=0, \quad i=1,2, \ldots .
$$

See Figures 1 and 2

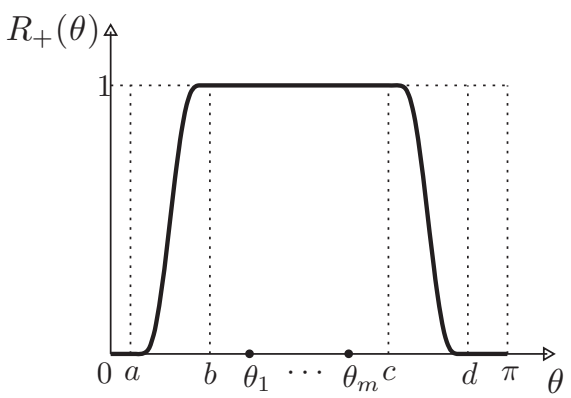

Figure 1. Graph of the the function $R_{+}(\theta)$ in (5.17). 


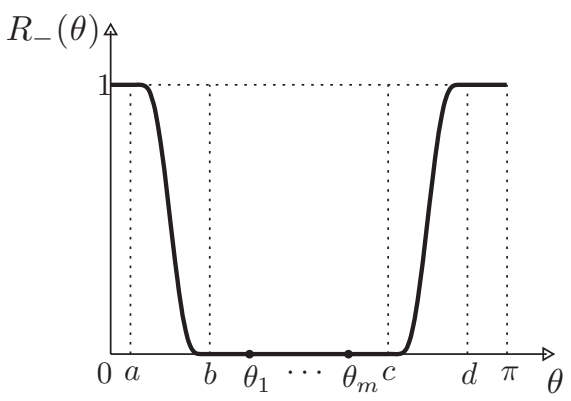

Figure 2. Graph of the the function $R_{-}(\theta)$ in (5.18).

5.2.2. Splitting $F(\theta)$ and $e_{n}[f]$. With the functions $R_{ \pm}(\theta)$ available, we now split $F(\theta)$ as in

$$
F(\theta)=F_{+}(\theta)+F_{-}(\theta) ; \quad F_{+}(\theta)=R_{+}(\theta) F(\theta), \quad F_{-}(\theta)=R_{-}(\theta) F(\theta) .
$$

Clearly,

$$
\begin{array}{llll}
F_{+}(\theta)=0 & \text { if } \theta<a \text { or } \theta>d, & F_{+}(\theta)=F(\theta) & \text { if } b<\theta<c, \\
F_{-}(\theta)=F(\theta) & \text { if } \theta<a \text { or } \theta>d, & F_{-}(\theta)=0 & \text { if } b<\theta<c .
\end{array}
$$

In addition,

$$
F_{ \pm}^{(i)}(a)=0=F_{ \pm}^{(i)}(d), \quad i=1,2, \ldots
$$

In view of (5.22) and (1.2),

$$
e_{n}[f]=e_{n}^{(+)}[f]+e_{n}^{(-)}[f], \quad e_{n}^{( \pm)}[f]=\widehat{n} \int_{-1}^{1} F_{ \pm}(\theta) P_{n}(\cos \theta) d \theta
$$

Going back to the variable $x$, it is easy to see that

$$
e_{n}^{( \pm)}[f]=\widehat{n} \int_{-1}^{1} f_{ \pm}(x) P_{n}(x) d x=e_{n}\left[f_{ \pm}\right], \quad f_{ \pm}(x)=\left.\frac{F_{ \pm}(\theta)}{\sin \theta}\right|_{\theta=\cos ^{-1} x} .
$$

To complete the proof, we need the asymptotic expansions for the integrals $e_{n}\left[f_{ \pm}\right]$, to which we turn next.

5.2.3. Completion of proof. We begin with $e_{n}\left[f_{-}\right]$. Clearly, $f_{-}(x)$ satisfies

$$
f_{-}(x)= \begin{cases}f(x) & \text { if } x<\cos d \text { or } x>\cos a, \\ 0 & \text { if } \cos c<x<\cos b,\end{cases}
$$

and, therefore, $f_{-} \in C^{\infty}(-1,1)$, and its asymptotic expansions as $x \rightarrow \pm 1$ are precisely those of $f(x)$. This implies that Theorem 3.1 applies to $e_{n}\left[f_{-}\right]$. Thus, as $n \rightarrow \infty, e_{n}\left[f_{-}\right]$has the asymptotic expansion

$$
e_{n}\left[f_{-}\right] \sim \sum_{\substack{s=0 \\ \alpha_{s}^{(+)} \notin \mathbb{Z}^{+}}}^{\infty} A_{s}^{(+)} \sum_{k=0}^{\infty} \frac{c_{k}\left(\alpha_{s}^{(+)}\right)}{\widehat{n}^{2\left(\alpha_{s}^{(+)}+k+1 / 2\right)}}+(-1)^{n} \sum_{\substack{s=0 \\ \alpha_{s}^{(-)} \notin \mathbb{Z}^{+}}}^{\infty} A_{s}^{(-)} \sum_{k=0}^{\infty} \frac{c_{k}\left(\alpha_{s}^{(-)}\right)}{\widehat{n}^{2\left(\alpha_{s}^{(-)}+k+1 / 2\right)}} .
$$


As for $e_{n}\left[f_{+}\right]$, we first observe that

$$
e_{n}\left[f_{+}\right]=\widehat{n} \sum_{r=0}^{m} I_{r, n}^{(+)} ; \quad I_{r, n}^{(+)}=\int_{\theta_{r}}^{\theta_{r+1}} F_{+}(\theta) P_{n}(\cos \theta) d \theta, \quad r=0,1, \ldots, m .
$$

In addition, by (5.23), we realize that

$$
\begin{aligned}
I_{0, n}^{(+)} & =\int_{a}^{\theta_{1}} F_{+}(\theta) P_{n}(\cos \theta) d \theta, \\
I_{r, n}^{(+)} & =\int_{\theta_{r}}^{\theta_{r+1}} F(\theta) P_{n}(\cos \theta) d \theta, \quad r=1, \ldots, m-1, \\
I_{m, n}^{(+)} & =\int_{\theta_{m}}^{d} F_{+}(\theta) P_{n}(\cos \theta) d \theta .
\end{aligned}
$$

It is clear that we can achieve our goal by determining the asymptotic expansion of each of the integrals $I_{r, n}^{(+)}$in (5.30) as $n \rightarrow \infty$. Recall that, for each $r \in\{1, \ldots, m-1\}$, the integrand of $I_{r, n}^{(+)}$, is in $C^{\infty}\left(\theta_{r}, \theta_{r+1}\right)$ and is singular both at $\theta_{r}$ and $\theta_{r+1}$, whereas that for $I_{0, n}^{(+)}$is singular only at $\theta_{1}$ and that for $I_{m, n}^{(+)}$is singular only at $\theta_{m}$. In addition, in all cases, the asymptotic expansions as $\theta \rightarrow \theta_{r} \pm$ of the respective integrands are precisely those of $F(\theta) P_{n}(\cos \theta)$. Therefore, Theorem 4.1 applies to each $I_{r, n}^{(+)}$.

Thus, for $I_{r, n}^{(+)}, 1 \leq r \leq m-1$, letting $r+1=r^{\prime}$, we have, as $n \rightarrow \infty$,

$$
\begin{aligned}
& I_{r, n}^{(+)} \sim e^{\mathrm{i} \widehat{n} \theta_{r}} \sum_{s=0}^{\infty} T_{r s}^{(+)} \sum_{\mu=0}^{\infty} \frac{G_{\mu}^{(+)}\left(\theta_{r} ; \gamma_{r s}^{(+)}\right)}{\widehat{n}_{r s}^{(+)}+\mu+3 / 2}+e^{-\mathrm{i} \widehat{n} \theta_{r}} \sum_{s=0}^{\infty} T_{r s}^{(+)} \sum_{\mu=0}^{\infty} \frac{\widehat{G}_{\mu}^{(+)}\left(\theta_{r} ; \gamma_{r s}^{(+)}\right)}{\widehat{n}_{r s}^{(+)}+\mu+3 / 2} \\
& +e^{\mathrm{i} \widehat{\mathrm{n}} \theta_{r^{\prime}}} \sum_{s=0}^{\infty} T_{r^{\prime} s}^{(-)} \sum_{\mu=0}^{\infty} \frac{G_{\mu}^{(-)}\left(\theta_{r^{\prime}} ; \gamma_{r^{\prime} s}^{(-)}\right)}{\widehat{n}^{\gamma_{r^{\prime} s}^{(-)}+\mu+3 / 2}}+e^{-\mathrm{i} \widehat{\mathrm{n}} \theta_{r^{\prime}}} \sum_{s=0}^{\infty} T_{r^{\prime} s}^{(-)} \sum_{\mu=0}^{\infty} \frac{\widehat{G}_{\mu}^{(-)}\left(\theta_{r^{\prime}} ; \gamma_{r^{\prime} s}^{(-)}\right)}{\widehat{n}_{r^{\prime} s}^{(-)}+\mu+3 / 2} .
\end{aligned}
$$

By (5.24), there is no contribution to $I_{0, n}^{(+)}$from $\theta=a$ and no contribution to $I_{m, n}^{(+)}$from $\theta=d$. Thus, as $n \rightarrow \infty$,

$$
\begin{aligned}
& I_{0, n}^{(+)} \sim e^{\mathrm{i} \hat{n} \theta_{1}} \sum_{s=0}^{\infty} T_{1 s}^{(-)} \sum_{\mu=0}^{\infty} \frac{G_{\mu}^{(-)}\left(\theta_{1} ; \gamma_{1 s}^{(-)}\right)}{\widehat{n}^{\gamma_{1 s}^{(-)}+\mu+3 / 2}} \\
& +e^{-\mathrm{i} \widehat{n} \theta_{1}} \sum_{s=0}^{\infty} T_{1 s}^{(-)} \sum_{\mu=0}^{\infty} \frac{\widehat{G}_{\mu}^{(-)}\left(\theta_{1} ; \gamma_{1 s}^{(-)}\right)}{\widehat{n}_{1 s}^{(-)}+\mu+3 / 2}, \\
& I_{m, n}^{(+)} \sim e^{\mathrm{i} \widehat{\widehat{n}} \theta_{m}} \sum_{s=0}^{\infty} T_{m s}^{(+)} \sum_{\mu=0}^{\infty} \frac{G_{\mu}^{(+)}\left(\theta_{m} ; \gamma_{m s}^{(+)}\right)}{\widehat{n}_{m s}^{(+)}+\mu+3 / 2} \\
& +e^{-\mathrm{i} \widehat{n} \theta_{m}} \sum_{s=0}^{\infty} T_{m s}^{(+)} \sum_{\mu=0}^{\infty} \frac{\widehat{G}_{\mu}^{(+)}\left(\theta_{m} ; \gamma_{m s}^{(-)}\right)}{\widehat{n}_{m s}^{(-)}+\mu+3 / 2} .
\end{aligned}
$$

Substituting (5.31)-(5.33) in (5.29), and adding (5.28), we obtain (4.8). This completes the proof. 


\section{Consequences of Theorem 4.2}

Theorem 4.2 concerns the general case in which $F(\theta)$ has arbitrary algebraic singularities in $[0, \pi]$, that is, the $\gamma_{r s}^{( \pm)}$in (2.4) and the $\alpha_{s}^{( \pm)}$in (2.7) are arbitrary. We now consider the special case in which $\gamma_{r s}^{( \pm)}=\gamma_{r}^{( \pm)}+s$, and $\alpha_{s}^{( \pm)}=\alpha^{( \pm)}+s$, $s=0,1, \ldots$. Then $\gamma_{r s}^{( \pm)}+\mu+1 / 2=\gamma_{r}^{( \pm)}+s+\mu+1 / 2$ and $\alpha_{s}^{( \pm)}+k+1 / 2=$ $\alpha^{( \pm)}+s+k+1 / 2$ in (4.8). Realizing now that $s+\mu$ and $s+k$ take on only the values $0,1,2, \ldots$, and re-expanding in negative powers of $n$ instead of $\widehat{n}$, and rearranging, we can rewrite (4.8) in the simpler form

$$
\begin{gathered}
e_{n}[f] \sim \sum_{r=1}^{m}\left\{e^{\mathrm{i} n \theta_{r}}\left[\sum_{s=0}^{\infty} \frac{L_{r s}^{(+)}}{n^{\gamma_{r}^{(+)}+s+1 / 2}}+\sum_{s=0}^{\infty} \frac{L_{r s}^{(-)}}{n^{\gamma_{r}^{(-)}+s+1 / 2}}\right]\right. \\
\left.+e^{-\mathrm{i} n \theta_{r}}\left[\sum_{s=0}^{\infty} \frac{\widehat{L}_{r s}^{(+)}}{n^{\gamma_{r}^{(+)}+s+1 / 2}}+\sum_{s=0}^{\infty} \frac{\widehat{L}_{r s}^{(-)}}{n^{\gamma_{r}^{(-)}+s+1 / 2}}\right]\right\} \\
+\sum_{s=0}^{\infty} \frac{M_{s}^{(+)}}{n^{2 \alpha(+)}+s+1}+(-1)^{n} \sum_{s=0}^{\infty} \frac{M_{s}^{(-)}}{n^{2 \alpha^{(-)}+s+1}}, \\
\text { where } M_{s}^{( \pm)}=0 \text { when } \alpha^{( \pm)} \in \mathbb{Z}^{+} .
\end{gathered}
$$

A further simplification takes place when $\gamma_{r}^{(+)}-\gamma_{r}^{(-)}$are all integers. Letting $\gamma_{r}=\min \left\{\gamma_{r}^{(+)}, \gamma_{r}^{(-)}\right\}$, (6.1) can be rewritten as in

$$
\begin{array}{r}
e_{n}[f] \sim \sum_{r=1}^{m}\left\{e^{\mathrm{i} n \theta_{r}} \sum_{s=0}^{\infty} \frac{L_{r s}}{n^{\gamma_{r}+s+1 / 2}}+e^{-\mathrm{i} n \theta_{r}} \sum_{s=0}^{\infty} \frac{\widehat{L}_{r s}}{n^{\gamma_{r}+s+1 / 2}}\right\} \\
+\sum_{s=0}^{\infty} \frac{M_{s}^{(+)}}{n^{2 \alpha^{(+)}+s+1}}+(-1)^{n} \sum_{s=0}^{\infty} \frac{M_{s}^{(-)}}{n^{2 \alpha^{(-)}+s+1}},
\end{array}
$$$$
\text { where } M_{s}^{( \pm)}=0 \text { when } \alpha^{( \pm)} \in \mathbb{Z}^{+} \text {. }
$$

This is the case when, for example, (i) $f(x)=(1-x)^{\alpha^{(+)}}(1+x)^{\alpha^{(-)}} g(x), g(x)$ being infinitely differentiable at $x= \pm 1$ and $g( \pm 1) \neq 0$, and (ii) $f(x)$ has finite jump discontinuities at $m$ interior points $x_{1}, \ldots, x_{m}$ of $(-1,1)$, with $f^{(k)}\left(x_{r} \pm\right)$ defined for all $k=0,1, \ldots$; in such a case, we will generally have $\gamma_{r}^{(+)}=\gamma_{r}^{(-)}=1$, and hence $\gamma_{r}=1$, for all $r$.

We now consider the problem of summing the infinite series $\sum_{n=0}^{\infty} e_{n}[f] P_{n}(x)$ that represents $f(x)$ for $x \in[-1,1]$. Multiplying the asymptotic expansion in (6.2) with that in (3.5) of Theorem 3.2. and rearranging, we realize that $e_{n}[f] P_{n}(\cos \theta)$ has an asymptotic expansion of the form

$$
e_{n}[f] P_{n}(\cos \theta) \sim \sum_{k=1}^{M} \zeta_{k}^{n} \sum_{i=0}^{\infty} \beta_{k i} n^{\delta_{k}-i} \quad \text { as } n \rightarrow \infty .
$$

where $M=4 m+4$ and the $\zeta_{k}$ (with corresponding $\delta_{k}$ ) are $e^{ \pm \mathrm{i}\left(\theta+\theta_{r}\right)}, e^{ \pm \mathrm{i}\left(\theta-\theta_{r}\right)}$, (with $\left.\delta_{k}=-\gamma_{r}-1\right), r=1, \ldots, m$, and $e^{ \pm \mathrm{i} \theta}$ (with $\left.\delta_{k}=-2 \alpha^{(+)}-3 / 2\right), e^{ \pm \mathrm{i}(\theta-\pi)}$ (with $\delta_{k}=-2 \alpha^{(-)}-3 / 2$ ). (Note that the $\zeta_{k}$ here are distinct and $\zeta_{k} \neq 1$.) This being the case the sequence $\left\{e_{n}[f] P_{n}(\cos \theta)\right\}_{n=0}^{\infty}$ belongs to the set of sequences denoted $\mathbf{b}^{(M)}$. See Sidi [11, Chapter 6, p. 123, Definition 6.1.2]. 
Since $\left|\zeta_{k}\right|=1$ for all $k$ in (6.3), the series $\sum_{n=0}^{\infty} e_{n}[f] P_{n}(x)$ converges very slowly for $-1<x<1$, especially when $f(x)$ has strong singularities on $[-1,1]$. To overcome this problem, we can apply suitable convergence acceleration methods to the sequence of partial sums of this series. The fact that the sequence $\left\{e_{n}[f] P_{n}(x)\right\}_{n=0}^{\infty}$ is in the class $\mathbf{b}^{(M)}$ suggests that the convergence of the infinite series $\sum_{n=0}^{\infty} e_{n}[f] P_{n}(x)$ can be accelerated very effectively by the $d^{(M)}$ transformation of Levin and Sidi [6]. See also [11, Chapter 6, p. 130, Definition 6.2.1]. Convergence acceleration can be achieved in this case also by the transformation of Shanks [8. See also [11, Chapter 16]. As a matter of fact, these two methods seem to be the only nonlinear methods that can achieve convergence acceleration on the series $\sum_{n=0}^{\infty} e_{n}[f] P_{n}(x)$ when $e_{n}[f]$ is as in (6.2). To apply these methods, we do not need to know the $\zeta_{k}$ and $\delta_{k}$ in (6.3). Mere knowledge of the existence of an asymptotic expansion of the form given in (6.3) is sufficient for deciding that these two transformations are suitable. Finally, these methods can be applied in

a much more economical way by introducing the $Q_{n}(x)$, the Legendre functions of the second kind, as has been shown in Sidi [10. See also [11, Chapter 13].

So far, we do not have any theoretical results on the performance of these methods on such series with $M>1$. We do, however, have rigorous results on the convergence and stability of the generalized Richardson extraplation process (GREP) of the author [9] on such problems (assuming that the $\zeta_{k}$ and $\delta_{k}$ are available), and these appear in the recent paper Sidi 13 .

\section{Appendix: Asymptotic expansion of Associated Legendre FUnCtions of THE FIRST AND SECOND KINDS}

In the sequel, $P_{\nu}^{\mu}(x)$ and $Q_{\nu}^{\mu}(x)$ denote the associated Legendre functions of degree $\nu$ and order $\mu$, of the first and second kinds, respectively. See, Abramowitz and Stegun [1, Chapter 8], for example.

Theorem A.1. Let $\mu$ and $\nu$ be real and $\mu<1 / 2$. There exist analytic functions $\phi_{k}(z)$ that are regular for $|z|=1, z \neq \pm 1$, such that

$$
Z_{\nu}^{\mu}(\cos \theta) \equiv P_{\nu}^{\mu}(\cos \theta)-\mathrm{i} \frac{2}{\pi} Q_{\nu}^{\mu}(\cos \theta) \sim e^{\mathrm{i} \widehat{\nu} \theta} \sum_{k=0}^{\infty} \frac{\phi_{k}\left(e^{\mathrm{i} \theta}\right)}{\widehat{\nu}^{k-\mu+1 / 2}} \quad \text { as } \nu \rightarrow \infty, \quad \widehat{\nu}=\nu+1 / 2,
$$

uniformly for $\epsilon \leq \theta \leq \pi-\epsilon$, with $\epsilon \in(0, \pi / 4)$ fixed. That is, for each $p=0,1, \ldots$, and for $0<\theta<\pi$, there holds

$$
Z_{\nu}^{\mu}(\cos \theta)=e^{\mathrm{i} \mathrm{i} \theta}\left[\sum_{k=0}^{p-1} \frac{\phi_{k}\left(e^{\mathrm{i} \theta}\right)}{\widehat{\nu}^{k-\mu+1 / 2}}+R_{p, \nu}(\theta)\right],
$$

where

$$
R_{p, \nu}(\theta)=O\left(\widehat{\nu}^{-p+\mu-1 / 2}\right) \quad \text { as } \nu \rightarrow \infty, \text { uniformly for } \epsilon \leq \theta \leq \pi-\epsilon .
$$

Actually, with $D_{\theta}=\frac{d}{d \theta}$,

$$
\begin{aligned}
\phi_{k}\left(e^{\mathrm{i} \theta}\right) & =(-1)^{k} \frac{2^{\mu+1}}{\pi^{1 / 2}}\left(\begin{array}{c}
\mu-1 / 2 \\
k
\end{array}\right)(\sin \theta)^{\mu} e^{\mathrm{i}[(\mu+1 / 2) \theta-\pi / 2]} \\
& \times B_{k}^{(\mu+1 / 2)}\left((\mathrm{i} / 2) D_{\theta}\right)\left[\left(1-e^{\mathrm{i} 2 \theta}\right)^{-\mu-1 / 2}\right] .
\end{aligned}
$$


Here, $B_{k}^{(\sigma)}(y)=\sum_{s=0}^{k}\left(\begin{array}{l}k \\ s\end{array}\right) B_{k-s}^{(\sigma)} y^{s}$ is the $k$ th generalized Bernoulli polynomial (see footnote 1), and, by $B_{k}^{(\sigma)}\left(c D_{\theta}\right) Y(\theta)$, we mean

$$
B_{k}^{(\sigma)}\left(c D_{\theta}\right) Y(\theta)=\sum_{s=0}^{k}\left(\begin{array}{l}
k \\
s
\end{array}\right) B_{k-s}^{(\sigma)} c^{s}\left[D_{\theta}^{s} Y(\theta)\right]=\sum_{s=0}^{k}\left(\begin{array}{l}
k \\
s
\end{array}\right) B_{k-s}^{(\sigma)} c^{s} Y^{(s)}(\theta) .
$$

Remark. It is clear that, when $\mu$ and $\nu$ are real,

$$
P_{\nu}^{\mu}(\cos \theta)=\Re Z_{\nu}^{\mu}(\cos \theta), \quad Q_{\nu}^{\mu}(\cos \theta)=-\frac{\pi}{2} \Im Z_{\nu}^{\mu}(\cos \theta) .
$$

In addition, invoking

$$
\phi_{0}\left(e^{\mathrm{i} \theta}\right)=\frac{2^{\mu+1}}{\pi^{1 / 2}}(\sin \theta)^{\mu} e^{\mathrm{i}[(\mu+1 / 2) \theta-\pi / 2]}\left(1-e^{\mathrm{i} 2 \theta}\right)^{-\mu-1 / 2},
$$

in (A.1), namely, in

$$
Z_{\nu}^{\mu}(\cos \theta)=e^{\mathrm{i} \hat{\nu} \theta}\left[\phi_{0}\left(e^{\mathrm{i} \theta}\right) \widehat{\nu}^{\mu-1 / 2}+O\left(\widehat{\nu}^{\mu-3 / 2}\right] \quad \text { as } \nu \rightarrow \infty,\right.
$$

after some simple manipulation, we obtain the asymptotic results given in [1, p. 336, Eqs. 8.10.7, 8.10.8].

Proof. We begin with the Fourier series representation of the associated Legendre functions $P_{\nu}^{\mu}(\cos \theta)$ and $Q_{\nu}^{\mu}(\cos \theta)$ given in Abramowitz and Stegun 11, p. 335, Eqs. 8.7.1, 8.7.2], which are valid for real $\mu$ and $\nu$ and $\mu<1 / 2$. It can easily be seen that we can express $Z_{\nu}^{\mu}(\cos \theta)$ in terms of the Gaussian hypergeometric function as follows:

$$
\begin{aligned}
Z_{\nu}^{\mu}(\cos \theta)= & \frac{2^{\mu+1} \Gamma(\nu+\mu+1)}{\pi^{1 / 2} \Gamma(\nu+3 / 2)}(\sin \theta)^{\mu} \\
& \times e^{\mathrm{i}[(\nu+\mu+1) \theta-\pi / 2]} F\left(\mu+\frac{1}{2}, \nu+\mu+1 ; \nu+\frac{3}{2} ; \zeta\right), \quad \zeta=e^{\mathrm{i} 2 \theta} .
\end{aligned}
$$

Invoking the integral representation of $F(a, b ; c ; z)$ given in [1 p. 558, Eq. 15.3.1], we obtain the following integral representation for $Z_{\nu}^{\mu}(\cos \theta)$ :

$$
\begin{aligned}
Z_{\nu}^{\mu}(\cos \theta) & =\frac{2^{\mu+1}(\sin \theta)^{\mu}}{\pi^{1 / 2} \Gamma(1 / 2-\mu)} \\
& \times e^{\mathrm{i}[(\nu+\mu+1) \theta-\pi / 2]} \int_{0}^{1} t^{\nu+\mu}(1-t)^{-\mu-1 / 2}(1-\zeta t)^{-\mu-1 / 2} d t, \quad \zeta=e^{\mathrm{i} 2 \theta} .
\end{aligned}
$$

Upon making the change of variable $t=e^{-\tau}$, (A.6) becomes

$$
Z_{\nu}^{\mu}(\cos \theta)=\frac{2^{\mu+1}(\sin \theta)^{\mu}}{\pi^{1 / 2} \Gamma(1 / 2-\mu)} e^{\mathrm{i}[(\nu+\mu+1) \theta-\pi / 2]} \int_{0}^{\infty} e^{-\widehat{\nu} \tau} \tau^{-\mu-1 / 2} g(\tau ; \zeta) d \tau,
$$

where

$$
g(\tau ; \zeta)=u(\tau) v(\tau ; \zeta) ; \quad u(\tau)=\left(\frac{\tau}{e^{\tau}-1}\right)^{\mu+1 / 2}, \quad v(\tau ; \zeta)=\left(1-\zeta e^{-\tau}\right)^{-\mu-1 / 2} .
$$

Now, when $\epsilon \leq \theta \leq \pi-\epsilon, g \in C^{\infty}[0, \infty)$ as a function of $\tau$ and has a (convergent) Maclaurin series

$$
g(\tau ; \zeta)=\sum_{k=0}^{\infty} g^{(k)}(0 ; \zeta) \frac{\tau^{k}}{k !} ; \quad g^{(k)}(\tau ; \zeta)=\frac{d^{k}}{d \tau^{k}} g(\tau ; \zeta) .
$$


[Here and in what follows, $w^{(k)}(\tau)=\frac{d^{k}}{d \tau^{k}} w(\tau)$.] We can now apply Watson's lemma (see, Olver [7, for example) to the integral in (A.7), and obtain the asymptotic expansion

$$
\int_{0}^{\infty} e^{-\widehat{\nu} \tau} \tau^{-\mu-1 / 2} g(\tau ; \zeta) d \tau \sim \sum_{k=0}^{\infty} \frac{g^{(k)}(0 ; \zeta)}{k !} \frac{\Gamma(k-\mu+1 / 2)}{\widehat{\nu}^{k-\mu+1 / 2}} \quad \text { as } \nu \rightarrow \infty .
$$

Upon substituting (A.10) in A.7), and recalling that $\zeta=e^{\mathrm{i} 2 \theta}$, we obtain (A.1), with

$$
\phi_{k}\left(e^{\mathrm{i} \theta}\right)=\frac{2^{\mu+1} \Gamma(k-\mu+1 / 2)}{\pi^{1 / 2} \Gamma(1 / 2-\mu)}(\sin \theta)^{\mu} e^{\mathrm{i}[(\mu+1 / 2) \theta-\pi / 2]} \frac{g^{(k)}\left(0 ; e^{\mathrm{i} 2 \theta}\right)}{k !} .
$$

To obtain a closed-form expression for $g^{(k)}\left(0 ; e^{\mathrm{i} 2 \theta}\right)$, we express $g(\tau ; \zeta)$ as the Cauchy product of the Maclaurin series of the functions $u(\tau)$ and $v(\tau ; \zeta)$ in (A.8). First, we have

$$
u(\tau)=\sum_{k=0}^{\infty} B_{k}^{(\mu+1 / 2)} \frac{\tau^{k}}{k !} .
$$

Next, by the fact that $\tau \geq 0$, for $|w|<1$ and arbitrary $\alpha$, we have

$$
\begin{aligned}
\left(1-w e^{-\tau}\right)^{\alpha} & =\sum_{s=0}^{\infty}(-1)^{s}\left(\begin{array}{c}
\alpha \\
s
\end{array}\right) w^{s} e^{-s \tau}=\sum_{s=0}^{\infty}(-1)^{s}\left(\begin{array}{l}
\alpha \\
s
\end{array}\right) w^{s} \sum_{k=0}^{\infty}(-1)^{k} s^{k} \frac{\tau^{k}}{k !} \\
& =\sum_{k=0}^{\infty}(-1)^{k} \frac{\tau^{k}}{k !} \sum_{s=0}^{\infty}(-1)^{s}\left(\begin{array}{l}
\alpha \\
s
\end{array}\right) s^{k} w^{s}=\sum_{k=0}^{\infty}(-1)^{k}\left[\left(w \frac{d}{d w}\right)^{k}(1-w)^{\alpha}\right] \frac{\tau^{k}}{k !} .
\end{aligned}
$$

Since $\left(1-w e^{-\tau}\right)^{\alpha}$ is analytic for all $w, w \notin[1,+\infty)$, the last equality holds also when $|w|=1$, but $w \neq 1$. From this, we have

$$
\begin{aligned}
v(\tau ; \zeta) & =\sum_{k=0}^{\infty}(-1)^{k}\left[\left(\zeta \frac{d}{d \zeta}\right)^{k}(1-\zeta)^{-\mu-1 / 2}\right] \frac{\tau^{k}}{k !} \\
& =\sum_{k=0}^{\infty}\left[\frac{d^{k}}{d \theta^{k}}\left(1-e^{\mathrm{i} 2 \theta}\right)^{-\mu-1 / 2}\right] \frac{(\mathrm{i} \tau / 2)^{k}}{k !} .
\end{aligned}
$$

Combining now (A.12) and (A.13) and (A.8), we obtain

$$
\begin{aligned}
\frac{1}{k !} g^{(k)}\left(0 ; e^{\mathrm{i} 2 \theta}\right) & =\sum_{s=0}^{k} \frac{B_{k-s}^{(\mu+1 / 2)}}{(k-s) !} \frac{(\mathrm{i} / 2)^{s}}{s !}\left[\frac{d^{s}}{d \theta^{s}}\left(1-e^{\mathrm{i} 2 \theta}\right)^{-\mu-1 / 2}\right] . \\
& =\frac{1}{k !} B_{k}^{(u+1 / 2)}\left((i / 2) D_{\theta}\right)\left[\left(1-e^{i 2 \theta}\right)^{-\mu-1 / 2}\right] .
\end{aligned}
$$

The explicit form of $\phi_{k}\left(e^{\mathrm{i} \theta}\right)$ given in (A.4) can now be obtained by substituting (A.14) in (A.11), and by invoking the facts that

$$
\frac{\Gamma(z+k)}{\Gamma(z)}=(z)_{k} \quad \text { and } \quad \frac{(z)_{k}}{k !}=(-1)^{k}\left(\begin{array}{c}
-z \\
k
\end{array}\right) .
$$

To prove that (A.1) holds uniformly for $\epsilon \leq \theta \leq \pi-\epsilon$, we proceed as follows: By first expanding $u(\tau)$ in powers of $e^{-\tau}$, and then, by differentiating the resulting (convergent) expansion with respect to $\tau$, it can be shown that

$$
u^{(k)}(\tau)=O\left(\tau^{\mu+1 / 2} e^{-(\mu+1 / 2) \tau}\right) \quad \text { as } \tau \rightarrow \infty, \quad k=0,1, \ldots .
$$


By this and by the fact that $u(\tau)$ is infinitely differentiable for $\tau \geq 0$, we conclude that $u_{k}(\tau)$ is of exponential order and hence

$$
\left|u^{(k)}(\tau)\right| \leq U_{k} e^{\lambda \tau}, \quad \text { for } \tau \geq 0, \quad k=0,1, \ldots,
$$

where $U_{k}$ are some positive constants and $\lambda>-\mu-1 / 2$. Next, by the fact that $v(\tau ; \zeta)$ is infinitely differentiable for $\tau \geq 0$ and

$$
v^{(k)}(\tau ; \zeta)=\sum_{i=0}^{k} w_{k i}\left(1-\zeta e^{-\tau}\right)^{-\mu-1 / 2-i}, \quad \text { for some constants } w_{k i},
$$

and because

$$
|v(\tau ; \zeta)| \leq\left\{\begin{array}{ll}
(\sin 2 \epsilon)^{-\mu-1 / 2} & \text { if } \mu>-1 / 2, \\
2^{-\mu-1 / 2} & \text { if } \mu \leq-1 / 2,
\end{array} \text { for } \tau \geq 0 \text { and } \epsilon \leq \theta \leq \pi-\epsilon\right.
$$

we also have that

$$
\left|v^{(k)}(\tau ; \zeta)\right| \leq V_{k}, \quad \text { for } \tau \geq 0 \text { and } \epsilon \leq \theta \leq \pi-\epsilon, \quad k=0,1, \ldots
$$

where $V_{k}$ are some positive constants. Finally, by (A.15) and (A.16) and by

$$
g^{(k)}(\tau ; \zeta)=\sum_{s=0}^{k}\left(\begin{array}{l}
k \\
s
\end{array}\right) u^{(k-s)}(\tau) v^{(s)}(\tau ; \zeta)
$$

we conclude that

$$
\left|g^{(k)}(\tau ; \zeta)\right| \leq G_{k} e^{\lambda \tau}, \quad \text { for } \tau \geq 0 \text { and } \epsilon \leq \theta \leq \pi-\epsilon, \quad k=0,1, \ldots,
$$

where $G_{k}$ are some positive constants and $\lambda$ is as in (A.15).

Let us now replace $g(\tau ; \zeta)$ in A.7 by its Maclaurin series with remainder, namely,

$$
\begin{aligned}
g(\tau ; \zeta) & =\sum_{k=0}^{p-1} g^{(k)}(0 ; \zeta) \frac{\tau^{k}}{k !}+S_{p}(\tau ; \zeta) \frac{\tau^{p}}{p !} \\
S_{p}(\tau ; \zeta) & =\left[\Re g^{(p)}\left(\widehat{\tau}_{R} ; \zeta\right)+\mathrm{i} \Im g^{(p)}\left(\widehat{\tau}_{I} ; \zeta\right)\right] \text { for some } \widehat{\tau}_{R}, \widehat{\tau}_{I} \in(0, \tau) .
\end{aligned}
$$

(Note that $\widehat{\tau}_{R}$ and $\widehat{\tau}_{I}$ depend on $\tau$ and $\zeta$.) It is clear by that $\left|S_{p}(\tau ; \zeta)\right| \leq$ $C_{p} e^{\lambda \tau}$ for all $\tau \in[0, \infty)$ and $\theta \in[\epsilon, \pi-\epsilon], C_{p}$ being some positive constant. Carrying out the individual integrals, and noting that, for all $\nu>\lambda$,

$$
\begin{aligned}
\left|\int_{0}^{\infty} e^{-\widehat{\nu} \tau} \tau^{-\mu-1 / 2} S_{p}(\tau ; \zeta) \frac{\tau^{p}}{p !} d \tau\right| & \leq \frac{C_{p}}{p !} \int_{0}^{\infty} e^{-(\widehat{\nu}-\lambda) \tau} \tau^{p-\mu-1 / 2} d \tau \\
& =\frac{C_{p}}{p !} \frac{\Gamma(p-\mu+1 / 2)}{(\widehat{\nu}-\lambda)^{p-\mu+1 / 2}}
\end{aligned}
$$

we see that (A.2) holds with (A.3) and aniformly for $\epsilon \leq \theta \leq \pi-\epsilon$. This completes the proof.

\section{ACKNOWLEDGEMENTS}

The author would like to thank Eran Treister for producing the graphs in Figures 1 and 2. He would like to also thank the anonymous referee for drawing his attention to references [4] and [5]. 


\section{REFERENCES}

1. M. Abramowitz and I.A. Stegun, Handbook of mathematical functions with formulas, graphs, and mathematical tables, Nat. Bur. Standards Appl. Math. Series, no. 55, US Government Printing Office, Washington, D.C., 1964. MR0167642 (29:4914)

2. G.E. Andrews, R. Askey, and R. Roy, Special functions, Cambridge University Press, Cambridge, 1999. MR1688958 (2000g:33001)

3. N. Bleistein and R.A. Handelsman, Asymptotic expansions of integrals, Holt, Rinehart and Winston, New York, 1975.

4. T.T. Cîrulis, Asymptotic representation of the Fourier coefficients of functions in series of Legendre polynomials (Russian), Latviǔsk. Mat. Ežegodnik Vyp. 19 (1976), 47-62, 243-244.

5. M.K. Jain and M.M. Chawla, The estimation of the coefficients in the Legendre series expansion of a function, J. Math. Phys. Sci. 1 (1967), 247-260. MR0244695 (39:6009)

6. D. Levin and A. Sidi, Two new classes of nonlinear transformations for accelerating the convergence of infinite integrals and series, Appl. Math. Comp. 9 (1981), 175-215. Originally appeared as a Tel Aviv University preprint in 1975. MR650681 (83d:65010)

7. F.W.J. Olver, Asymptotics and special functions, Academic Press, New York, 1974. MR 0435697 (55:8655)

8. D. Shanks, Nonlinear transformations of divergent and slowly convergent sequences, J. Math. and Phys. 34 (1955), 1-42. MR0068901 (16:961e)

9. A. Sidi, Some properties of a generalization of the Richardson extrapolation process, J. Inst. Maths. Applics. 24 (1979), 327-346. MR550478 (81a:65011)

10. Acceleration of convergence of (generalized) Fourier series by the d-transformation, Annals Numer. Math. 2 (1995), 381-406. MR1343544 (96h:65005)

11. __ Practical extrapolation methods: Theory and applications, Cambridge Monographs on Applied and Computational Mathematics, no. 10, Cambridge University Press, Cambridge, 2003. MR.1994507 (2004e:65005)

12. _ Asymptotic expansions of Legendre series coefficients for functions with endpoint singularities, Asymptot. Anal. 65 (2009), 175-190. MR2574341

13. __ Asymptotic analysis of a generalized Richardson extrapolation process on linear sequences, Math. Comp. 79 (2010), 1681-1695.

14. _ A simple approach to asymptotic expansions for Fourier integrals of singular functions, Appl. Math. Comput. 216 (2010), 3378-3385. MR2653157

15. G. Szegö, Orthogonal polynomials, fourth ed., American Mathematical Society Colloquium Publications, Volume 23, American Mathematical Society, Providence, Rhode Island, 1975. MR0372517(51:8724)

Computer Science Department, Technion, Israel Institute of Technology, Haifa 32000, ISRAEL

E-mail address: asidi@cs.technion.ac.il

$U R L:$ http://www.cs.technion.ac.il/〜asidi/ 